\title{
ON MODEL OF MANUFACTURING OF AN INTEGRATED CIRCUITS BASED ON HETERO- STRUCTURES UNDER INFLUENCE OF MISMATCH-INDUCED STRESS AND POROSITY OF MATERIALS
}

\section{E. L. PANKRATOV}

Nizhny Novgorod State University

23 Gagarin Avenue

Nizhny Novgorod, 603950

Russia

Nizhny Novgorod State Technical University

24 Minin Street

Nizhny Novgorod, 603950

Russia

e-mail: elp2004@mail.ru

Keywords and phrases: downconversion mixer circuit, optimization of manufacturing, increasing of element integration rate.

Received July 30, 2020

(C) 2021 Scientific Advances Publishers

This work is licensed under the Creative Commons Attribution International License (CC BY 3.0).

http://creativecommons.org/licenses/by/3.0/deed.en_US

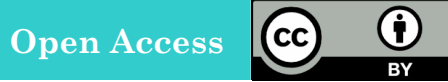




\begin{abstract}
In this paper, we introduce an approach to increase density of field-effect transistors framework a downconversion mixer circuit. Framework the approach we consider manufacturing the mixer in heterostructure with specific configuration. Several required areas of the heterostructure should be doped by diffusion or ion implantation. After that dopant and radiation defects should be annealed by framework optimized scheme. We also consider an approach to decrease value of mismatch-induced stress in the considered heterostructure. We introduce an analytical approach to analyze mass and heat transport in heterostructures during manufacturing of integrated circuits with account mismatch-induced stress.
\end{abstract}

\title{
1. Introduction
}

In the present time several actual problems of the solid state electronics (such as increasing of performance, reliability and density of elements of integrated circuits: diodes, field-effect and bipolar transistors) are intensively solving [1-6]. To increase the performance of these devices it is attracted an interest determination of materials with higher values of charge carriers mobility [7-10]. One way to decrease dimensions of elements of integrated circuits is manufacturing them in thin film heterostructures [3-5, 11]. In this case, it is possible to use inhomogeneity of heterostructure and necessary optimization of doping of electronic materials [12] and development of epitaxial technology to improve these materials (including analysis of mismatch-induced stress) [13-15]. An alternative approaches to increase dimensions of integrated circuits are using of laser and microwave types of annealing [16-18].

Framework the paper we introduce an approach to manufacture fieldeffect transistors. The approach gives a possibility to decrease their dimensions with increasing their density framework a downconversion mixer circuit. We also consider possibility to decrease mismatch-induced stress to decrease quantity of defects, generated due to the stress. In this paper we consider a heterostructure, which consist of a substrate and an epitaxial layer (see Figure 1). We also consider a buffer layer between the substrate and the epitaxial layer. The epitaxial layer includes into itself several sections, which were manufactured by using another materials. These sections have been doped by diffusion or ion implantation to manufacture the required types of conductivity ( $p$ or $n$ ). These areas became sources, drains and gates (see Figure 1). After this doping it is 
required annealing of dopant and/or radiation defects. Main aim of the present paper is analysis of redistribution of dopant and radiation defects to determine conditions, which correspond to decreasing of elements of the considered voltage reference and at the same time to increase their density. At the same time we consider a possibility to decrease mismatchinduced stress.

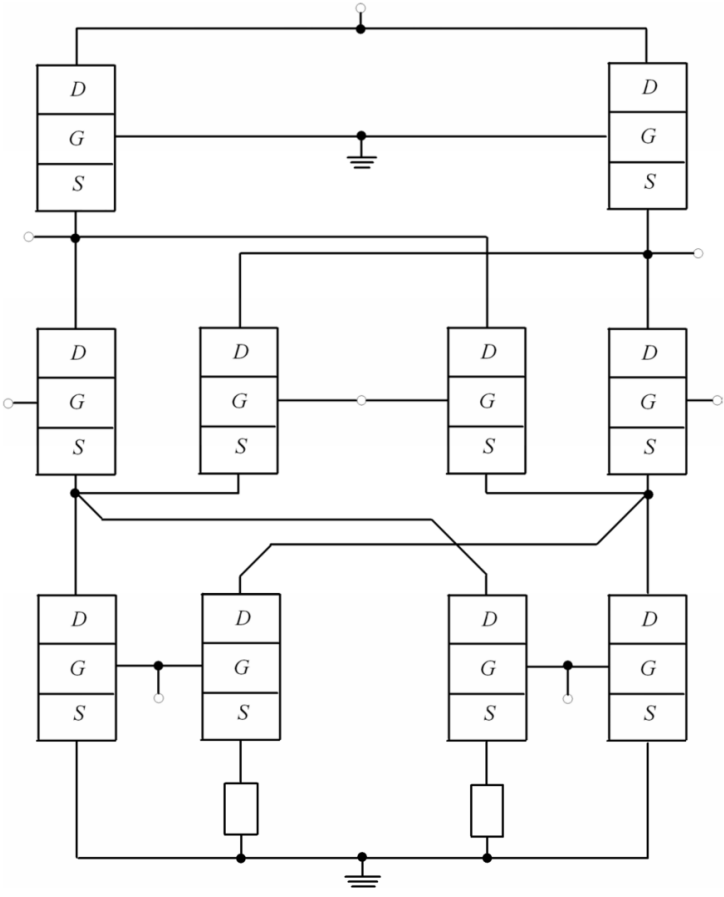

Figure 1(a). Structure of the considered mixer [19].

\begin{tabular}{|c|}
\hline Epitaxial layer \\
\hline Buffer layer \\
\hline Substrate \\
\\
\hline
\end{tabular}

Figure 1(b). Heterostructure with a substrate, epitaxial layers and buffer layer (view from side). 


\section{Method of Solution}

To solve our aim we determine and analyzed spatio-temporal distribution of concentration of dopant in the considered heterostructure. We determine the distribution by solving the second Fick's law in the following form [1, 20-23]:

$$
\begin{aligned}
\frac{\partial C(x, y, z, t)}{\partial t}= & \frac{\partial}{\partial x}\left[D \frac{\partial C(x, y, z, t)}{\partial x}\right] \\
& +\frac{\partial}{\partial y}\left[D \frac{\partial C(x, y, z, t)}{\partial y}\right]+\frac{\partial}{\partial z}\left[D \frac{\partial C(x, y, z, t)}{\partial z}\right] \\
& +\Omega \frac{\partial}{\partial x}\left[\frac{D_{S}}{k T} \nabla_{S} \mu_{1}(x, y, z, t) \int_{0}^{L_{z}} C(x, y, W, t) d W\right] \\
& +\Omega \frac{\partial}{\partial y}\left[\frac{D_{S}}{k T} \nabla_{S} \mu_{1}(x, y, z, t) \int_{0}^{L_{z}} C(x, y, W, t) d W\right] \\
& +\frac{\partial}{\partial x}\left[\frac{D_{C S}}{\bar{V} k T} \frac{\partial \mu_{2}(x, y, z, t)}{\partial x}\right]+\frac{\partial}{\partial y}\left[\frac{D_{C S}}{\bar{V} k T} \frac{\partial \mu_{2}(x, y, z, t)}{\partial y}\right] \\
& +\frac{\partial}{\partial z}\left[\frac{D_{C S}}{\bar{V} k T} \frac{\partial \mu_{2}(x, y, z, t)}{\partial z}\right],
\end{aligned}
$$

with boundary and initial conditions

$$
\begin{gathered}
\left.\frac{\partial C(x, y, z, t)}{\partial x}\right|_{x=0}=0,\left.\frac{\partial C(x, y, z, t)}{\partial x}\right|_{x=L_{x}}=0,\left.\frac{\partial C(x, y, z, t)}{\partial y}\right|_{y=0}=0, \\
\left.\frac{\partial C(x, y, z, t)}{\partial y}\right|_{y=L_{y}}=0,\left.\frac{\partial C(x, y, z, t)}{\partial z}\right|_{z=0}=0,\left.\frac{\partial C(x, y, z, t)}{\partial z}\right|_{z=L_{z}}=0, \\
C(x, y, z, 0)=f_{C}(x, y, z) .
\end{gathered}
$$

Here $C(x, y, z, t)$ is the spatio-temporal distribution of concentration of dopant; $\Omega$ is the atomic volume of dopant; $\nabla_{s}$ is the symbol of surficial 
gradient; $\int_{0}^{L_{z}} C(x, y, z, t) d z$ is the surficial concentration of dopant on interface between layers of heterostructure (in this situation we assume, that $Z$-axis is perpendicular to interface between layers of heterostructure); $\mu_{1}(x, y, z, t)$ and $\mu_{2}(x, y, z, t)$ are the chemical potential due to the presence of mismatch-induced stress and porosity of material; $D$ and $D_{S}$ are the coefficients of volumetric and surficial diffusions. Values of dopant diffusions coefficients depends on properties of materials of heterostructure, speed of heating and cooling of materials during annealing and spatio-temporal distribution of concentration of dopant. Dependences of dopant diffusions coefficients on parameters could be approximated by the following relations [24-26]:

$$
\begin{aligned}
D_{C}= & D_{L}(x, y, z, T)\left[1+\xi \frac{C^{\gamma}(x, y, z, t)}{P^{\gamma}(x, y, z, T)}\right] \\
& \times\left[1+\varsigma_{1} \frac{V(x, y, z, t)}{V^{*}}+\varsigma_{2} \frac{V^{2}(x, y, z, t)}{\left(V^{*}\right)^{2}}\right], \\
D_{S}= & D_{S L}(x, y, z, T)\left[1+\xi_{S} \frac{C^{\gamma}(x, y, z, t)}{P^{\gamma}(x, y, z, T)}\right] \\
& \times\left[1+\varsigma_{1} \frac{V(x, y, z, t)}{V^{*}}+\varsigma_{2} \frac{V^{2}(x, y, z, t)}{\left(V^{*}\right)^{2}}\right] .
\end{aligned}
$$

Here $D_{L}(x, y, z, T)$ and $D_{L S}(x, y, z, T)$ are the spatial (due to accounting all layers of heterostructure) and temperature (due to Arrhenius law) dependences of dopant diffusion coefficients; $T$ is the temperature of annealing; $P(x, y, z, T)$ is the limit of solubility of dopant; parameter $\gamma$ depends on properties of materials and could be integer in the following interval $\gamma \in[1,3]$ [24]; $V(x, y, z, t)$ is the spatiotemporal distribution of concentration of radiation vacancies; $V^{*}$ is the equilibrium distribution of vacancies. Concentrational dependence of 
dopant diffusion coefficient has been described in details in [24]. Spatiotemporal distributions of concentration of point radiation defects have been determined by solving the following system of equations [20-23, 25, 26]:

$$
\begin{aligned}
& \frac{\partial I(x, y, z, t)}{\partial t}=\frac{\partial}{\partial x}\left[D_{I}(x, y, z, T) \frac{\partial I(x, y, z, t)}{\partial x}\right] \\
& +\frac{\partial}{\partial y}\left[D_{I}(x, y, z, T) \frac{\partial I(x, y, z, t)}{\partial y}\right] \\
& +\frac{\partial}{\partial z}\left[D_{I}(x, y, z, T) \frac{\partial I(x, y, z, t)}{\partial z}\right] \\
& -k_{I, I}(x, y, z, T) I^{2}(x, y, z, t) \\
& -k_{I, V}(x, y, z, T) I(x, y, z, t) V(x, y, z, t) \\
& +\Omega \frac{\partial}{\partial x}\left[\frac{D_{I S}}{k T} \nabla_{S} \mu(x, y, z, t) \int_{0}^{L_{z}} I(x, y, W, t) d W\right] \\
& +\Omega \frac{\partial}{\partial y}\left[\frac{D_{I S}}{k T} \nabla_{S} \mu(x, y, z, t) \int_{0}^{L_{z}} I(x, y, W, t) d W\right] \\
& +\frac{\partial}{\partial x}\left[\frac{D_{I S}}{\overline{V k} T} \frac{\partial \mu_{2}(x, y, z, t)}{\partial x}\right]+\frac{\partial}{\partial y}\left[\frac{D_{I S}}{\overline{V k} T} \frac{\partial \mu_{2}(x, y, z, t)}{\partial y}\right] \\
& +\frac{\partial}{\partial z}\left[\frac{D_{I S}}{\overline{V k} T} \frac{\partial \mu_{2}(x, y, z, t)}{\partial z}\right] \\
& \frac{\partial V(x, y, z, t)}{\partial t}=\frac{\partial}{\partial x}\left[D_{V}(x, y, z, T) \frac{\partial V(x, y, z, t)}{\partial x}\right] \\
& +\frac{\partial}{\partial y}\left[D_{V}(x, y, z, T) \frac{\partial V(x, y, z, t)}{\partial y}\right] \\
& +\frac{\partial}{\partial z}\left[D_{V}(x, y, z, T) \frac{\partial V(x, y, z, t)}{\partial z}\right]
\end{aligned}
$$




$$
\begin{aligned}
& -k_{V, V}(x, y, z, T) V^{2}(x, y, z, t) \\
& -k_{I, V}(x, y, z, T) I(x, y, z, t) V(x, y, z, t), \\
& +\Omega \frac{\partial}{\partial x}\left[\frac{D_{V S}}{k T} \nabla_{S} \mu(x, y, z, t) \int_{0}^{L_{z}} V(x, y, W, t) d W\right] \\
& +\Omega \frac{\partial}{\partial y}\left[\frac{D_{V S}}{k T} \nabla_{S} \mu(x, y, z, t) \int_{0}^{L_{z}} V(x, y, W, t) d W\right] \\
& +\frac{\partial}{\partial x}\left[\frac{D_{V S}}{\overline{V k} T} \frac{\partial \mu_{2}(x, y, z, t)}{\partial x}\right]+\frac{\partial}{\partial y}\left[\frac{D_{V S}}{\overline{V k} T} \frac{\partial \mu_{2}(x, y, z, t)}{\partial y}\right] \\
& +\frac{\partial}{\partial z}\left[\frac{D_{V S}}{\overline{V k} T} \frac{\partial \mu_{2}(x, y, z, t)}{\partial z}\right],
\end{aligned}
$$

with boundary and initial conditions

$$
\begin{gathered}
\left.\frac{\partial I(x, y, z, t)}{\partial x}\right|_{x=0}=0,\left.\quad \frac{\partial I(x, y, z, t)}{\partial x}\right|_{x=L_{x}}=0,\left.\quad \frac{\partial I(x, y, z, t)}{\partial y}\right|_{y=0}=0, \\
\left.\frac{\partial I(x, y, z, t)}{\partial y}\right|_{y=L_{y}}=0,\left.\quad \frac{\partial I(x, y, z, t)}{\partial z}\right|_{z=0}=0,\left.\quad \frac{\partial I(x, y, z, t)}{\partial z}\right|_{z=L_{z}}=0, \\
\left.\frac{\partial V(x, y, z, t)}{\partial x}\right|_{x=0}=0,\left.\quad \frac{\partial V(x, y, z, t)}{\partial x}\right|_{x=L_{x}}=0,\left.\quad \frac{\partial V(x, y, z, t)}{\partial y}\right|_{y=0}=0, \\
\left.\frac{\partial V(x, y, z, t)}{\partial y}\right|_{y=L_{y}}=0,\left.\quad \frac{\partial V(x, y, z, t)}{\partial z}\right|_{z=0}=0,\left.\quad \frac{\partial V(x, y, z, t)}{\partial z}\right|_{z=L_{z}}=0, \\
I(x, y, z, 0)=f_{I}(x, y, z), \quad V(x, y, z, 0)=f_{V}(x, y, z), \\
V\left(x_{1}+V_{n} t, y_{1}+V_{n} t, z_{1}+V_{n} t, t\right)=V_{\infty}\left(1+\frac{2 \ell \omega}{k T \sqrt{x_{1}^{2}+y_{1}^{2}+z_{1}^{2}}}\right) .
\end{gathered}
$$


Here $I(x, y, z, t)$ is the spatio-temporal distribution of concentration of radiation interstitials; $I^{*}$ is the equilibrium distribution of interstitials; $D_{I}(x, y, z, T), D_{V}(x, y, z, T), D_{I S}(x, y, z, T), D_{V S}(x, y, z, T)$ are the coefficients of volumetric and surficial diffusions of interstitials and vacancies, respectively; terms $V^{2}(x, y, z, t)$ and $I^{2}(x, y, z, t)$ correspond to generation of divacancies and diinterstitials, respectively (see, for example, [26] and appropriate references in this book); $k_{I, V}(x, y, z, T), k_{I, I}(x, y, z, T)$ and $k_{V, V}(x, y, z, T)$ are the parameters of recombination of point radiation defects and generation of their complexes; $k$ is the Boltzmann constant; $\omega=a^{3}, a$ is the interatomic distance; $\ell$ is the specific surface energy. To account porosity of buffer layers we assume, that porous are approximately cylindrical with average values $r=\sqrt{x_{1}^{2}+y_{1}^{2}}$ and $z_{1}$ before annealing [23]. With time small pores decomposing on vacancies. The vacancies absorbing by larger pores [27]. With time large pores became larger due to absorbing the vacancies and became more spherical [27]. Distribution of concentration of vacancies in heterostructure, existing due to porosity, could be determined by summing on all pores, i.e.,

$V(x, y, z, t)=\sum_{i=0}^{l} \sum_{j=0}^{m} \sum_{k=0}^{n} V_{p}(x+i \alpha, y+j \beta, z+k \chi, t), R=\sqrt{x^{2}+y^{2}+z^{2}}$.

Here $\alpha, \beta$ and $\chi$ are the average distances between centers of pores in directions $x, y$ and $z ; l, m$ and $n$ are the quantity of pores inappropriate directions.

Spatio-temporal distributions of divacancies $\Phi_{V}(x, y, z, t)$ and diinterstitials $\Phi_{I}(x, y, z, t)$ could be determined by solving the following system of equations $[25,26]$ : 


$$
\begin{aligned}
\frac{\partial \Phi_{I}(x, y, z, t)}{\partial t}= & \frac{\partial}{\partial x}\left[D_{\Phi_{I}}(x, y, z, T) \frac{\partial \Phi_{I}(x, y, z, t)}{\partial x}\right] \\
& +\frac{\partial}{\partial y}\left[D_{\Phi_{I}}(x, y, z, T) \frac{\partial \Phi_{I}(x, y, z, t)}{\partial y}\right] \\
& +\frac{\partial}{\partial z}\left[D_{\Phi_{I}}(x, y, z, T) \frac{\partial \Phi_{I}(x, y, z, t)}{\partial z}\right] \\
& +\Omega \frac{\partial}{\partial x}\left[\frac{D_{\Phi_{I} S}}{k T} \nabla_{S} \mu_{1}(x, y, z, t) \int_{0}^{L_{z}} \Phi_{I}(x, y, W, t) d W\right] \\
& +\Omega \frac{\partial}{\partial y}\left[\frac{D_{\Phi_{I} S}}{k T} \nabla_{S} \mu_{1}(x, y, z, t) \int_{0}^{L_{z}} \Phi_{I}(x, y, W, t) d W\right] \\
& +k_{I, I}(x, y, z, T) I^{2}(x, y, z, t)+\frac{\partial}{\partial x}\left[\frac{D_{\Phi_{I} S}}{\bar{V} k T} \frac{\partial \mu_{2}(x, y, z, t)}{\partial x}\right] \\
& +\frac{\partial}{\partial y}\left[\frac{D_{\Phi_{I} S}}{\bar{V} k T} \frac{\partial \mu_{2}(x, y, z, t)}{\partial y}\right]+\frac{\partial}{\partial z}\left[\frac{D_{\Phi_{I} S}}{\bar{V} k T} \frac{\partial \mu_{2}(x, y, z, t)}{\partial z}\right] \\
& +k_{I}(x, y, z, T) I(x, y, z, t) ;
\end{aligned}
$$$$
\frac{\partial \Phi_{V}(x, y, z, t)}{\partial t}=\frac{\partial}{\partial x}\left[D_{\Phi_{V}}(x, y, z, T) \frac{\partial \Phi_{V}(x, y, z, t)}{\partial x}\right]
$$$$
+\frac{\partial}{\partial y}\left[D_{\Phi_{V}}(x, y, z, T) \frac{\partial \Phi_{V}(x, y, z, t)}{\partial y}\right]
$$$$
+\frac{\partial}{\partial z}\left[D_{\Phi_{V}}(x, y, z, T) \frac{\partial \Phi_{V}(x, y, z, t)}{\partial z}\right]
$$$$
+\Omega \frac{\partial}{\partial x}\left[\frac{D_{\Phi_{V} S}}{k T} \nabla_{S} \mu_{1}(x, y, z, t) \int_{0}^{L_{z}} \Phi_{V}(x, y, W, t) d W\right]
$$ 


$$
\begin{aligned}
& +\Omega \frac{\partial}{\partial y}\left[\frac{D_{\Phi_{V} S}}{k T} \nabla_{S} \mu_{1}(x, y, z, t) \int_{0}^{L_{z}} \Phi_{V}(x, y, W, t) d W\right] \\
& +k_{V, V}(x, y, z, T) V^{2}(x, y, z, t)+\frac{\partial}{\partial x}\left[\frac{D_{\Phi_{V} S}}{\bar{V} k T} \frac{\partial \mu_{2}(x, y, z, t)}{\partial x}\right] \\
& +\frac{\partial}{\partial y}\left[\frac{D_{\Phi_{V} S}}{\bar{V} k T} \frac{\partial \mu_{2}(x, y, z, t)}{\partial y}\right]+\frac{\partial}{\partial z}\left[\frac{D_{\Phi_{V} S}}{\bar{V} k T} \frac{\partial \mu_{2}(x, y, z, t)}{\partial z}\right] \\
& +k_{V}(x, y, z, T) V(x, y, z, t),
\end{aligned}
$$

with boundary and initial conditions

$$
\begin{gathered}
\left.\frac{\partial \Phi_{I}(x, y, z, t)}{\partial x}\right|_{x=0}=0,\left.\frac{\partial \Phi_{I}(x, y, z, t)}{\partial x}\right|_{x=L_{x}}=0,\left.\frac{\partial \Phi_{I}(x, y, z, t)}{\partial y}\right|_{y=0}=0 \\
\left.\frac{\partial \Phi_{I}(x, y, z, t)}{\partial y}\right|_{y=L_{y}}=0,\left.\frac{\partial \Phi_{I}(x, y, z, t)}{\partial z}\right|_{z=0}=0,\left.\frac{\partial \Phi_{I}(x, y, z, t)}{\partial z}\right|_{z=L_{z}}=0 \\
\left.\frac{\partial \Phi_{V}(x, y, z, t)}{\partial x}\right|_{x=0}=0,\left.\frac{\partial \Phi_{V}(x, y, z, t)}{\partial x}\right|_{x=L_{x}}=0,\left.\frac{\partial \Phi_{V}(x, y, z, t)}{\partial y}\right|_{y=0}=0 \\
\left.\frac{\partial \Phi_{V}(x, y, z, t)}{\partial y}\right|_{y=L_{y}}=0,\left.\frac{\partial \Phi_{V}(x, y, z, t)}{\partial z}\right|_{z=0}=0,\left.\frac{\partial \Phi_{V}(x, y, z, t)}{\partial z}\right|_{z=L_{z}}=0 \\
\Phi_{I}(x, y, z, 0)=f_{\Phi I}(x, y, z), \Phi_{V}(x, y, z, 0)=f_{\Phi V}(x, y, z) .
\end{gathered}
$$

Here $\quad D_{\Phi I}(x, y, z, T), D_{\Phi V}(x, y, z, T), D_{\Phi I S}(x, y, z, T), \quad$ and $D_{\Phi V S}(x, y, z, T)$ are the coefficients of volumetric and surficial diffusions of complexes of radiation defects; $k_{I}(x, y, z, T)$ and $k_{V}(x, y, z, T)$ are the parameters of decay of complexes of radiation defects. 
Chemical potential $\mu_{1}$ in Equation (1) could be determine by the following relation [20]:

$$
\mu_{1}=E(z) \Omega \sigma_{i j}\left[u_{i j}(x, y, z, t)+u_{j i}(x, y, z, t)\right] / 2,
$$

where $E(z)$ is the Young modulus, $\sigma_{i j}$ is the stress tensor; $u_{i j}=\frac{1}{2}$ $\left(\frac{\partial u_{i}}{\partial x_{j}}+\frac{\partial u_{j}}{\partial x_{i}}\right)$ is the deformation tensor; $u_{i}, u_{j}$ are the components $u_{x}(x, y, z, t), u_{y}(x, y, z, t)$ and $u_{z}(x, y, z, t)$ of the displacement vector $\vec{u}(x, y, z, t) ; x_{i}, x_{j}$ are the coordinate $x, y, z$. The Equation (3) could be transform to the following form:

$$
\begin{aligned}
\mu(x, y, z, t)= & {\left[\frac{\partial u_{i}(x, y, z, t)}{\partial x_{j}}+\frac{\partial u_{j}(x, y, z, t)}{\partial x_{i}}\right] } \\
& \times\left\{\frac{1}{2}\left[\frac{\partial u_{i}(x, y, z, t)}{\partial x_{j}}+\frac{\partial u_{j}(x, y, z, t)}{\partial x_{i}}\right]\right. \\
& -\varepsilon_{0} \delta_{i j}+\frac{\sigma(z) \delta_{i j}}{1-2 \sigma(z)}\left[\frac{\partial u_{k}(x, y, z, t)}{\partial x_{k}}-3 \varepsilon_{0}\right] \\
& \left.-K(z) \beta(z)\left[T(x, y, z, t)-T_{0}\right] \delta_{i j}\right\} \frac{\Omega}{2} E(z),
\end{aligned}
$$

where $\sigma$ is Poisson coefficient; $\varepsilon_{0}=\left(a_{s}-a_{E L}\right) / a_{E L}$ is the mismatch parameter; $a_{s}, a_{E L}$ are lattice distances of the substrate and the epitaxial layer; $K$ is the modulus of uniform compression; $\beta$ is the coefficient of thermal expansion; $T_{r}$ is the equilibrium temperature, which coincide (for our case) with room temperature. Components of displacement vector could be obtained by solution of the following equations [21]: 
$\rho(z) \frac{\partial^{2} u_{x}(x, y, z, t)}{\partial t^{2}}=\frac{\partial \sigma_{x x}(x, y, z, t)}{\partial x}+\frac{\partial \sigma_{x y}(x, y, z, t)}{\partial y}+\frac{\partial \sigma_{x z}(x, y, z, t)}{\partial z}$

$\rho(z) \frac{\partial^{2} u_{y}(x, y, z, t)}{\partial t^{2}}=\frac{\partial \sigma_{y x}(x, y, z, t)}{\partial x}+\frac{\partial \sigma_{y y}(x, y, z, t)}{\partial y}+\frac{\partial \sigma_{y z}(x, y, z, t)}{\partial z}$,

$\rho(z) \frac{\partial^{2} u_{z}(x, y, z, t)}{\partial t^{2}}=\frac{\partial \sigma_{z x}(x, y, z, t)}{\partial x}+\frac{\partial \sigma_{z y}(x, y, z, t)}{\partial y}+\frac{\partial \sigma_{z z}(x, y, z, t)}{\partial z}$

where $\sigma_{i j}=\frac{E(z)}{2[1+\sigma(z)]}\left[\frac{\partial u_{i}(x, y, z, t)}{\partial x_{j}}+\frac{\partial u_{j}(x, y, z, t)}{\partial x_{i}}-\frac{\delta_{i j}}{3} \frac{\partial u_{k}(x, y, z, t)}{\partial x_{k}}\right]$

$+K(z) \delta_{i j} \frac{\partial u_{k}(x, y, z, t)}{\partial x_{k}}-\beta(z) K(z)\left[T(x, y, z, t)-T_{r}\right], \rho(z)$ is the density of materials of heterostructure, $\delta_{i j}$ is the Kronecker symbol. With account the relation for $\sigma_{i j}$ last system of equation could be written as

$$
\begin{aligned}
\rho(z) \frac{\partial^{2} u_{x}(x, y, z, t)}{\partial t^{2}}= & \left\{K(z)+\frac{5 E(z)}{6[1+\sigma(z)]}\right\} \frac{\partial^{2} u_{x}(x, y, z, t)}{\partial x^{2}} \\
& +\left\{K(z)-\frac{E(z)}{3[1+\sigma(z)]}\right\} \frac{\partial^{2} u_{y}(x, y, z, t)}{\partial x \partial y} \\
& +\frac{E(z)}{2[1+\sigma(z)]}\left[\frac{\partial^{2} u_{y}(x, y, z, t)}{\partial y^{2}}+\frac{\partial^{2} u_{z}(x, y, z, t)}{\partial z}\right] \\
& +\left[K(z)+\frac{E(z)}{3[1+\sigma(z)]}\right] \frac{\partial^{2} u_{z}(x, y, z, t)}{\partial x \partial z} \\
& -K(z) \beta(z) \frac{\partial T(x, y, z, t)}{\partial x} ;
\end{aligned}
$$




$$
\begin{aligned}
& \rho(z) \frac{\partial^{2} u_{y}(x, y, z, t)}{\partial t^{2}}=\frac{E(z)}{2[1+\sigma(z)]}\left[\frac{\partial^{2} u_{y}(x, y, z, t)}{\partial x^{2}}+\frac{\partial^{2} u_{x}(x, y, z, t)}{\partial x \partial y}\right] \\
& -\frac{\partial T(x, y, z, t)}{\partial y} K(z) \beta(z) \\
& +\frac{\partial}{\partial z}\left\{\frac{E(z)}{2[1+\sigma(z)]}\left[\frac{\partial u_{y}(x, y, z, t)}{\partial z}+\frac{\partial u_{z}(x, y, z, t)}{\partial y}\right]\right\} \\
& +\frac{\partial^{2} u_{y}(x, y, z, t)}{\partial y^{2}}\left\{\frac{5 E(z)}{12[1+\sigma(z)]}+K(z)\right\} \\
& +\left\{K(z)-\frac{E(z)}{6[1+\sigma(z)]}\right\} \frac{\partial^{2} u_{y}(x, y, z, t)}{\partial y \partial z} \\
& +K(z) \frac{\partial^{2} u_{y}(x, y, z, t)}{\partial x \partial y} \\
& \rho(z) \frac{\partial^{2} u_{z}(x, y, z, t)}{\partial t^{2}}=\frac{E(z)}{2[1+\sigma(z)]}\left[\frac{\partial^{2} u_{z}(x, y, z, t)}{\partial x^{2}}+\frac{\partial^{2} u_{z}(x, y, z, t)}{\partial y^{2}}\right. \\
& \left.+\frac{\partial^{2} u_{x}(x, y, z, t)}{\partial x \partial z}+\frac{\partial^{2} u_{y}(x, y, z, t)}{\partial y \partial z}\right] \\
& +\frac{\partial}{\partial z}\left\{K ( z ) \left[\frac{\partial u_{x}(x, y, z, t)}{\partial x}+\frac{\partial u_{y}(x, y, z, t)}{\partial y}\right.\right. \\
& \left.\left.+\frac{\partial u_{x}(x, y, z, t)}{\partial z}\right]\right\}+\frac{1}{6} \frac{\partial}{\partial z}\left\{\frac { E ( z ) } { 1 + \sigma ( z ) } \left[6 \frac{\partial u_{z}(x, y, z, t)}{\partial z}\right.\right. \\
& \left.\left.-\frac{\partial u_{x}(x, y, z, t)}{\partial x}-\frac{\partial u_{y}(x, y, z, t)}{\partial y}-\frac{\partial u_{z}(x, y, z, t)}{\partial z}\right]\right\} \\
& -K(z) \beta(z) \frac{\partial T(x, y, z, t)}{\partial z} .
\end{aligned}
$$


Conditions for the system of Equation (8) could be written in the form

$$
\begin{gathered}
\frac{\partial \vec{u}(0, y, z, t)}{\partial x}=0 ; \frac{\partial \vec{u}\left(L_{x}, y, z, t\right)}{\partial x}=0 ; \frac{\partial \vec{u}(x, 0, z, t)}{\partial y}=0 ; \\
\frac{\partial \vec{u}\left(x, L_{y}, z, t\right)}{\partial y}=0 ; \frac{\partial \vec{u}(x, y, 0, t)}{\partial z}=0 ; \frac{\partial \vec{u}\left(x, y, L_{z}, t\right)}{\partial z}=0 ; \\
\vec{u}(x, y, z, 0)=\vec{u}_{0} ; \vec{u}(x, y, z, \infty)=\vec{u}_{0} .
\end{gathered}
$$

We determine spatio-temporal distributions of concentrations of dopant and radiation defects by solving the Equations (1), (3) and (5) framework standard method of averaging of function corrections [28]. Previously we transform the Equations (1), (3) and (5) to the following form with account initial distributions of the considered concentrations:

$$
\begin{aligned}
\frac{\partial C(x, y, z, t)}{\partial t}= & \frac{\partial}{\partial x}\left[D \frac{\partial C(x, y, z, t)}{\partial x}\right]+\frac{\partial}{\partial y}\left[D \frac{\partial C(x, y, z, t)}{\partial y}\right] \\
& +\frac{\partial}{\partial z}\left[D \frac{\partial C(x, y, z, t)}{\partial z}\right]+f_{C}(x, y, z) \delta(t) \\
& +\frac{\partial}{\partial x}\left[\frac{D_{C S}}{\bar{V} k T} \frac{\partial \mu_{2}(x, y, z, t)}{\partial x}\right]+\frac{\partial}{\partial y}\left[\frac{D_{C S}}{\bar{V} k T} \frac{\partial \mu_{2}(x, y, z, t)}{\partial y}\right] \\
& +\frac{\partial}{\partial z}\left[\frac{D_{C S}}{\bar{V} k T} \frac{\partial \mu_{2}(x, y, z, t)}{\partial z}\right] \\
& +\Omega \frac{\partial}{\partial x}\left[\frac{D_{S}}{k T} \nabla_{S} \mu(x, y, z, t) \int_{0}^{L_{z}} C(x, y, W, t) d W\right] \\
& +\Omega \frac{\partial}{\partial y}\left[\frac{D_{S}}{k T} \nabla_{S} \mu(x, y, z, t) \int_{0}^{L_{z}} C(x, y, W, t) d W\right]
\end{aligned}
$$




$$
\begin{aligned}
\frac{\partial I(x, y, z, t)}{\partial t}= & \frac{\partial}{\partial x}\left[D_{I}(x, y, z, T) \frac{\partial I(x, y, z, t)}{\partial x}\right] \\
& +\frac{\partial}{\partial y}\left[D_{I}(x, y, z, T) \frac{\partial I(x, y, z, t)}{\partial y}\right] \\
& +\frac{\partial}{\partial z}\left[D_{I}(x, y, z, T) \frac{\partial I(x, y, z, t)}{\partial z}\right] \\
& +\Omega \frac{\partial}{\partial x}\left[\frac{D_{I S}}{k T} \nabla_{S} \mu_{1}(x, y, z, t) \int_{0}^{L_{z}} I(x, y, W, t) d W\right] \\
& +\Omega \frac{\partial}{\partial y}\left[\frac{D_{I S}}{k T} \nabla_{S} \mu_{1}(x, y, z, t) \int_{0}^{L_{z}} I(x, y, W, t) d W\right] \\
& -k_{I, I}(x, y, z, T) I^{2}(x, y, z, t) \\
& -k_{I, V}(x, y, z, T) I(x, y, z, t) V(x, y, z, t)+f_{I}(x, y, z) \delta(t) ; \\
& -k_{I, V}(x, y, z, T) I(x, y, z, t) V(x, y, z, t)+f_{V}(x, y, z) \delta(t), \\
& +\Omega \frac{\partial}{\partial x}\left[D_{V}(x, y, z, T) \frac{\partial V(x, y, z, t)}{\partial x}\right] \\
& +\frac{\partial}{\partial y}\left[\frac{D_{V S}}{k T} \nabla_{S} \mu_{1}(x, y, z, t) \int_{0}^{L_{z}} V(x, y, W, t) d W\right] \\
& +\frac{\partial}{\partial z}\left[D_{V}(x, y, z, T) \frac{\partial V(x, y, z, t)}{\partial y}\right]
\end{aligned}
$$




$$
\begin{aligned}
& \frac{\partial \Phi_{I}(x, y, z, t)}{\partial t}=\frac{\partial}{\partial x}\left[D_{\Phi_{I}}(x, y, z, T) \frac{\partial \Phi_{I}(x, y, z, t)}{\partial x}\right] \\
& +\frac{\partial}{\partial y}\left[D_{\Phi_{I}}(x, y, z, T) \frac{\partial \Phi_{I}(x, y, z, t)}{\partial y}\right] \\
& +\frac{\partial}{\partial z}\left[D_{\Phi_{I}}(x, y, z, T) \frac{\partial \Phi_{I}(x, y, z, t)}{\partial z}\right] \\
& +\Omega \frac{\partial}{\partial x}\left[\frac{D_{\Phi_{I}} S}{k T} \nabla_{S} \mu_{1}(x, y, z, t) \int_{0}^{L_{z}} \Phi_{I}(x, y, W, t) d W\right] \\
& +\Omega \frac{\partial}{\partial y}\left[\frac{D_{\Phi_{I} S}}{k T} \nabla_{S} \mu_{1}(x, y, z, t) \int_{0}^{L_{z}} \Phi_{I}(x, y, W, t) d W\right] \\
& +k_{I}(x, y, z, T) I(x, y, z, t)+\frac{\partial}{\partial x}\left[\frac{D_{\Phi_{I} S}}{\bar{V} k T} \frac{\partial \mu_{2}(x, y, z, t)}{\partial x}\right] \\
& +\frac{\partial}{\partial y}\left[\frac{D_{\Phi_{I} S}}{\bar{V} k T} \frac{\partial \mu_{2}(x, y, z, t)}{\partial y}\right]+\frac{\partial}{\partial z}\left[\frac{D_{\Phi_{I} S}}{\bar{V} k T} \frac{\partial \mu_{2}(x, y, z, t)}{\partial z}\right] \\
& +k_{I, I}(x, y, z, T) I^{2}(x, y, z, t)+f_{\Phi_{I}}(x, y, z) \delta(t) ; \\
& \frac{\partial \Phi_{V}(x, y, z, t)}{\partial t}=\frac{\partial}{\partial x}\left[D_{\Phi_{V}}(x, y, z, T) \frac{\partial \Phi_{V}(x, y, z, t)}{\partial x}\right] \\
& +\frac{\partial}{\partial y}\left[D_{\Phi_{V}}(x, y, z, T) \frac{\partial \Phi_{V}(x, y, z, t)}{\partial y}\right] \\
& +\frac{\partial}{\partial z}\left[D_{\Phi_{V}}(x, y, z, T) \frac{\partial \Phi_{V}(x, y, z, t)}{\partial z}\right] \\
& +\Omega \frac{\partial}{\partial x}\left[\frac{D_{\Phi_{V} S}}{k T} \nabla_{S} \mu_{1}(x, y, z, t) \int_{0}^{L_{z}} \Phi_{V}(x, y, W, t) d W\right]
\end{aligned}
$$




$$
\begin{aligned}
& +\Omega \frac{\partial}{\partial y}\left[\frac{D_{\Phi_{V} S}}{k T} \nabla_{S} \mu_{1}(x, y, z, t) \int_{0}^{L_{z}} \Phi_{V}(x, y, W, t) d W\right] \\
& +k_{V}(x, y, z, T) V(x, y, z, t)+\frac{\partial}{\partial x}\left[\frac{D_{\Phi_{V} S}}{\bar{V} k T} \frac{\partial \mu_{2}(x, y, z, t)}{\partial x}\right] \\
& +\frac{\partial}{\partial y}\left[\frac{D_{\Phi_{V}}}{\bar{V} k T} \frac{\partial \mu_{2}(x, y, z, t)}{\partial y}\right]+\frac{\partial}{\partial z}\left[\frac{D_{\Phi_{V} S}}{\bar{V} k T} \frac{\partial \mu_{2}(x, y, z, t)}{\partial z}\right] \\
& +k_{V, V}(x, y, z, T) V^{2}(x, y, z, t)+f_{\Phi_{V}}(x, y, z) \delta(t)
\end{aligned}
$$

Farther we replace concentrations of dopant and radiation defects in right sides of Equations (1a), (3a) and (5a) on their not yet known average values $\alpha_{1 \rho}$. In this situation, we obtain equations for the first-order approximations of the required concentrations in the following form:

$$
\begin{aligned}
\frac{\partial C_{1}(x, y, z, t)}{\partial t}= & \alpha_{1 C} \Omega \frac{\partial}{\partial x}\left[z \frac{D_{S}}{k T} \nabla_{S} \mu_{1}(x, y, z, t)\right] \\
& +\alpha_{1 C} \Omega \frac{\partial}{\partial y}\left[z \frac{D_{S}}{k T} \nabla_{S} \mu_{1}(x, y, z, t)\right] \\
& +f_{C}(x, y, z) \delta(t)+\frac{\partial}{\partial x}\left[\frac{D_{C S}}{\bar{V} k T} \frac{\partial \mu_{2}(x, y, z, t)}{\partial x}\right] \\
& +\frac{\partial}{\partial y}\left[\frac{D_{C S}}{\bar{V} k T} \frac{\partial \mu_{2}(x, y, z, t)}{\partial y}\right]+\frac{\partial}{\partial z}\left[\frac{D_{C S}}{\bar{V} k T} \frac{\partial \mu_{2}(x, y, z, t)}{\partial z}\right],
\end{aligned}
$$




$$
\begin{aligned}
& \frac{\partial I_{1}(x, y, z, t)}{\partial t}=\alpha_{1 I} z \Omega \frac{\partial}{\partial x}\left[\frac{D_{I S}}{k T} \nabla_{S} \mu(x, y, z, t)\right] \\
& +\alpha_{1 I} \Omega \frac{\partial}{\partial y}\left[z \frac{D_{I S}}{k T} \nabla_{S} \mu(x, y, z, t)\right] \\
& +\frac{\partial}{\partial x}\left[\frac{D_{I S}}{\bar{V} k T} \frac{\partial \mu_{2}(x, y, z, t)}{\partial x}\right]+\frac{\partial}{\partial y}\left[\frac{D_{I S}}{\bar{V} k T} \frac{\partial \mu_{2}(x, y, z, t)}{\partial y}\right] \\
& +\frac{\partial}{\partial z}\left[\frac{D_{I S}}{\bar{V} k T} \frac{\partial \mu_{2}(x, y, z, t)}{\partial z}\right]+f_{I}(x, y, z) \delta(t) \\
& -\alpha_{1 I}^{2} k_{I, I}(x, y, z, T)-\alpha_{1 I} \alpha_{1 V} k_{I, V}(x, y, z, T) ; \\
& \frac{\partial V_{1}(x, y, z, t)}{\partial t}=\alpha_{1 V} z \Omega \frac{\partial}{\partial x}\left[\frac{D_{V S}}{k T} \nabla_{S} \mu_{1}(x, y, z, t)\right] \\
& +\alpha_{1 V} \Omega \frac{\partial}{\partial y}\left[z \frac{D_{V S}}{k T} \nabla_{S} \mu_{1}(x, y, z, t)\right] \\
& +\frac{\partial}{\partial x}\left[\frac{D_{V S}}{\bar{V} k T} \frac{\partial \mu_{2}(x, y, z, t)}{\partial x}\right]+\frac{\partial}{\partial y}\left[\frac{D_{V S}}{\bar{V} k T} \frac{\partial \mu_{2}(x, y, z, t)}{\partial y}\right] \\
& +\frac{\partial}{\partial z}\left[\frac{D_{V S}}{\bar{V} k T} \frac{\partial \mu_{2}(x, y, z, t)}{\partial z}\right]+f_{V}(x, y, z) \delta(t) \\
& -\alpha_{1 V}^{2} k_{V, V}(x, y, z, T)-\alpha_{1 I} \alpha_{1 V} k_{I, V}(x, y, z, T), \\
& \frac{\partial \Phi_{1 I}(x, y, z, t)}{\partial t}=\alpha_{1 \Phi_{I}} z \Omega \frac{\partial}{\partial x}\left[\frac{D_{\Phi_{I} S}}{k T} \nabla_{S} \mu_{1}(x, y, z, t)\right] \\
& +\alpha_{1 \Phi_{I}} z \Omega \frac{\partial}{\partial y}\left[\frac{D_{\Phi_{I}} S}{k T} \nabla_{S} \mu_{1}(x, y, z, t)\right] \\
& +\frac{\partial}{\partial x}\left[\frac{D_{\Phi_{I} S}}{\bar{V} k T} \frac{\partial \mu_{2}(x, y, z, t)}{\partial x}\right]+\frac{\partial}{\partial y}\left[\frac{D_{\Phi_{I}} S}{\bar{V} k T} \frac{\partial \mu_{2}(x, y, z, t)}{\partial y}\right]
\end{aligned}
$$




$$
\begin{aligned}
+ & \frac{\partial}{\partial z}\left[\frac{D_{\Phi_{I} S}}{\bar{V} k T} \frac{\partial \mu_{2}(x, y, z, t)}{\partial z}\right]+f_{\Phi_{I}}(x, y, z) \delta(t) \\
& +k_{I}(x, y, z, T) I(x, y, z, t) \\
& +k_{I, I}(x, y, z, T) I^{2}(x, y, z, t) ; \\
\frac{\partial \Phi_{1 V}(x, y, z, t)}{\partial t}= & \alpha_{1 \Phi_{V}} z \Omega \frac{\partial}{\partial x}\left[\frac{D_{\Phi_{V} S}}{k T} \nabla_{S} \mu_{1}(x, y, z, t)\right] \\
& +\alpha_{1 \Phi_{V}} z \Omega \frac{\partial}{\partial y}\left[\frac{D_{\Phi_{V} S}}{k T} \nabla_{S} \mu_{1}(x, y, z, t)\right] \\
& +\frac{\partial}{\partial x}\left[\frac{D_{\Phi_{V} S}}{\bar{V} k T} \frac{\partial \mu_{2}(x, y, z, t)}{\partial x}\right]+\frac{\partial}{\partial y}\left[\frac{D_{\Phi_{V} S}}{\bar{V} k T} \frac{\partial \mu_{2}(x, y, z, t)}{\partial y}\right] \\
& +\frac{\partial}{\partial z}\left[\frac{D_{\Phi_{V} S}}{\bar{V} k T} \frac{\partial \mu_{2}(x, y, z, t)}{\partial z}\right]+f_{\Phi_{V}}(x, y, z) \delta(t) \\
& +k_{V}(x, y, z, T) V(x, y, z, t) \\
& +k_{V, V}(x, y, z, T) V^{2}(x, y, z, t) .
\end{aligned}
$$

Integration of the left and right sides of the Equations (1b), (3b) and (5b) on time gives us possibility to obtain relations for above approximation in the final form

$$
\begin{aligned}
C_{1}(x, y, z, t)= & \alpha_{1 C} \Omega \frac{\partial}{\partial x} \int_{0}^{t} D_{S L}(x, y, z, T) \frac{z}{k T} \\
& \times\left[1+\varsigma_{1} \frac{V(x, y, z, \tau)}{V^{*}}+\varsigma_{2} \frac{V^{2}(x, y, z, \tau)}{\left(V^{*}\right)^{2}}\right] \\
& \left.\times \nabla_{S} \mu_{1}(x, y, z, \tau)\left[1+\frac{\xi_{S} \alpha_{1 C}^{\gamma}}{P^{\gamma}(x, y, z, T)}\right] d \tau\right\}
\end{aligned}
$$




$$
\begin{aligned}
& +\alpha_{1 C} \frac{\partial}{\partial y} \int_{0}^{t} D_{S L}(x, y, z, T)\left[1+\frac{\xi_{S} \alpha_{1 C}^{\gamma}}{P^{\gamma}(x, y, z, T)}\right] \\
& \times \Omega \nabla_{S} \mu_{1}(x, y, z, \tau) \frac{z}{k T}\left[1+\varsigma_{1} \frac{V(x, y, z, \tau)}{V^{*}}+\varsigma_{2} \frac{V^{2}(x, y, z, \tau)}{\left(V^{*}\right)^{2}}\right] d \tau \\
& +\frac{\partial}{\partial x} \int_{0}^{t} \frac{D_{C S}}{\bar{V} k T} \frac{\partial \mu_{2}(x, y, z, \tau)}{\partial x} d \tau+\frac{\partial}{\partial y} \int_{0}^{t} \frac{D_{C S}}{\bar{V} k T} \frac{\partial \mu_{2}(x, y, z, \tau)}{\partial y} d \tau \\
& +\frac{\partial}{\partial z} \int_{0}^{t} \frac{D_{C S}}{\bar{V} k T} \frac{\partial \mu_{2}(x, y, z, \tau)}{\partial z} d \tau+f_{C}(x, y, z), \\
& I_{1}(x, y, z, t)=\alpha_{1 I} z \Omega \frac{\partial}{\partial x} \int_{0}^{t} \frac{D_{I S}}{k T} \nabla_{S} \mu_{1}(x, y, z, \tau) d \tau \\
& +\alpha_{1 I} z \Omega \frac{\partial}{\partial y} \int_{0}^{t} \frac{D_{I S}}{k T} \nabla_{S} \mu_{1}(x, y, z, \tau) d \tau \\
& +\frac{\partial}{\partial x} \int_{0}^{t} \frac{D_{I S}}{\bar{V} k T} \frac{\partial \mu_{2}(x, y, z, t)}{\partial x} d \tau+\frac{\partial}{\partial y} \int_{0}^{t} \frac{D_{I S}}{\bar{V} k T} \frac{\partial \mu_{2}(x, y, z, t)}{\partial y} d \tau \\
& +\frac{\partial}{\partial z} \int_{0}^{t} \frac{D_{I S}}{\bar{V} k T} \frac{\partial \mu_{2}(x, y, z, t)}{\partial z} d \tau+f_{I}(x, y, z) \\
& -\alpha_{1 I}^{2} \int_{0}^{t} k_{I, I}(x, y, z, T) d \tau-\alpha_{1 I} \alpha_{1 V} \int_{0}^{t} k_{I, V}(x, y, z, T) d \tau
\end{aligned}
$$




$$
\begin{aligned}
V_{1}(x, y, z, t)= & \alpha_{1 V} z \Omega \frac{\partial}{\partial x} \int_{0}^{t} \frac{D_{V S}}{k T} \nabla_{S} \mu_{1}(x, y, z, \tau) d \tau \\
& +\alpha_{1 V} z \Omega \frac{\partial}{\partial y} \int_{0}^{t} \frac{D_{V S}}{k T} \nabla_{S} \mu_{1}(x, y, z, \tau) d \tau \\
& +\frac{\partial}{\partial x} \int_{0}^{t} \frac{D_{V S}}{\bar{V} k T} \frac{\partial \mu_{2}(x, y, z, t)}{\partial x} d \tau+\frac{\partial}{\partial y} \int_{0}^{t} \frac{D_{V S}}{\bar{V} k T} \frac{\partial \mu_{2}(x, y, z, t)}{\partial y} d \tau \\
& +\frac{\partial}{\partial z} \int_{0}^{t} \frac{D_{V S}}{\bar{V} k T} \frac{\partial \mu_{2}(x, y, z, t)}{\partial z} d \tau+f_{V}(x, y, z) \\
& -\alpha_{1 V}^{2} \int_{0}^{t} k_{V, V}(x, y, z, T) d \tau-\alpha_{1 I} \alpha_{1 V} \int_{0}^{t} k_{I, V}(x, y, z, T) d \tau
\end{aligned}
$$

$$
\begin{aligned}
\Phi_{1 I}(x, y, z, t)= & \alpha_{1 \Phi_{I}} z \Omega \frac{\partial}{\partial x} \int_{0}^{t} \frac{D_{\Phi_{I} S}}{k T} \nabla_{S} \mu_{1}(x, y, z, \tau) d \tau \\
& +\Omega \frac{\partial}{\partial x} \int_{0}^{t} \frac{D_{\Phi_{I} S}}{k T} \nabla_{S} \mu_{1}(x, y, z, \tau) d \tau \alpha_{1 \Phi_{I}} z+f_{\Phi_{I}}(x, y, z) \\
& +\frac{\partial}{\partial x} \int_{0}^{t} \frac{D_{\Phi_{I} S}}{\bar{V} k T} \frac{\partial \mu_{2}(x, y, z, \tau)}{\partial x} d \tau+\frac{\partial}{\partial y} \int_{0}^{t} \frac{D_{\Phi_{I} S}}{\bar{V} k T} \frac{\partial \mu_{2}(x, y, z, \tau)}{\partial y} d \tau \\
& +\frac{\partial}{\partial z} \int_{0}^{t} \frac{D_{\Phi_{I} S}}{\bar{V} k T} \frac{\partial \mu_{2}(x, y, z, \tau)}{\partial z} d \tau+\int_{0}^{t} k_{I}(x, y, z, T) I(x, y, z, \tau) d \tau \\
& +\int_{0}^{t} k_{I, I}(x, y, z, T) I^{2}(x, y, z, \tau) d \tau
\end{aligned}
$$




$$
\begin{aligned}
\Phi_{1 V}(x, y, z, t)= & \alpha_{1 \Phi_{V}} z \Omega \frac{\partial}{\partial x} \int_{0}^{t} \frac{D_{\Phi_{V} S}}{k T} \nabla_{S} \mu_{1}(x, y, z, \tau) d \tau \\
& +\Omega \frac{\partial}{\partial x} \int_{0}^{t} \frac{D_{\Phi_{V} S}}{k T} \nabla_{S} \mu_{1}(x, y, z, \tau) d \tau \alpha_{1 \Phi_{V}} z+f_{\Phi_{V}}(x, y, z) \\
& +\frac{\partial}{\partial x} \int_{0}^{t} \frac{D_{\Phi_{V} S}}{\bar{V} k T} \frac{\partial \mu_{2}(x, y, z, \tau)}{\partial x} d \tau+\frac{\partial}{\partial y} \int_{0}^{t} \frac{D_{\Phi_{V} S}}{\bar{V} k T} \frac{\partial \mu_{2}(x, y, z, \tau)}{\partial y} d \tau \\
& +\frac{\partial}{\partial z} \int_{0}^{t} \frac{D_{\Phi_{V} S}}{\bar{V} k T} \frac{\partial \mu_{2}(x, y, z, \tau)}{\partial z} d \tau+\int_{0}^{t} k_{V}(x, y, z, T) V(x, y, z, \tau) d \tau \\
& +\int_{0}^{t} k_{V, V}(x, y, z, T) V^{2}(x, y, z, \tau) d \tau .
\end{aligned}
$$

We determine average values of the first-order approximations of concentrations of dopant and radiation defects by the following standard relation [28]:

$$
\alpha_{1 \rho}=\frac{1}{\Theta L_{x} L_{y} L_{z}} \int_{0}^{\Theta} \int_{0}^{L_{x}} \int_{0}^{L_{y}} \int_{0}^{L_{z}} \rho_{1}(x, y, z, t) d z d y d x d t .
$$

Substitution of the relations (1c), (3c) and (5c) into relation (9) gives us possibility to obtain required average values in the following form:

$$
\begin{gathered}
\alpha_{1 C}=\frac{1}{L_{x} L_{y} L_{z}} \int_{0}^{L_{x}} \int_{0}^{L_{y}} \int_{0}^{L_{z}} f_{C}(x, y, z) d z d y d x, \\
\alpha_{1 I}=\sqrt{\frac{\left(a_{3}+A\right)^{2}}{4 a_{4}^{2}}-4\left(B+\frac{\left.\Theta a_{3} B+\Theta L_{x} L_{y} L_{z} a_{1}\right)}{a_{4}}\right)}-\frac{a_{3}+A}{4 a_{4}}, \\
\alpha_{1 V}=\frac{1}{S_{I V 00}}\left[\frac{\Theta}{\alpha_{1 I}} \int_{0}^{L_{x}} \int_{0}^{L_{y}} \int_{0}^{L_{z}} f_{I}(x, y, z) d z d y d x-\alpha_{1 I} S_{I I 00}-\Theta L_{x} L_{y} L_{z}\right],
\end{gathered}
$$


where

$$
\begin{aligned}
& S_{\rho \rho i j}=\int_{0}^{\Theta}(\Theta-t) \int_{0}^{L_{x}} \int_{0}^{L_{y}} \int_{0}^{L_{z}} k_{\rho, \rho}(x, y, z, T) I_{1}^{i}(x, y, z, t) V_{1}^{j}(x, y, z, t) d z d y d x d t \\
& a_{4}=S_{I I 00}\left(S_{I V 00}^{2}-S_{I I 00} S_{V V 00}\right), \\
& a_{3}=S_{I V 00} S_{I I 00}+S_{I V 00}^{2}-S_{I I 00} S_{V V 00}, \\
& a_{2}=\int_{0}^{L_{x}} \int_{0}^{L_{y}} \int_{0}^{L_{z}} f_{V}(x, y, z) d z d y d x \times S_{I V 00} S_{I V 00}^{2}+S_{I V 00} \Theta L_{x}^{2} L_{y}^{2} L_{z}^{2} \\
& +2 S_{V V 00} S_{I I 00} \int_{0}^{L_{x}} \int_{0}^{L_{y}} \int_{0}^{L_{z}} f_{I}(x, y, z) d z d y d x \\
& -\Theta L_{x}^{2} L_{y}^{2} L_{z}^{2} S_{V V 00}-S_{I V 00}^{2} \int_{0}^{L_{x}} \int_{0}^{L_{y}} \int_{0}^{L_{z}} f_{I}(x, y, z) d z d y d x, \\
& a_{1}=S_{I V 00} \int_{0}^{L_{x}} \int_{0}^{L_{y}} \int_{0}^{L_{z}} f_{I}(x, y, z) d z d y d x \\
& a_{0}=S_{V V 00}\left[\int_{0}^{L_{x}} \int_{0}^{L_{y}} \int_{0}^{L_{z}} f_{I}(x, y, z) d z d y d x\right]^{2}, A=\sqrt{8 y+\Theta^{2} \frac{a_{3}^{2}}{a_{4}^{2}}-4 \Theta \frac{a_{2}}{a_{4}}}, \\
& B=\frac{\Theta a_{2}}{6 a_{4}}+\sqrt[3]{\sqrt{q^{2}+p^{3}-q}}-\sqrt[3]{\sqrt{q^{2}+p^{3}+q}}, \\
& q=\frac{\Theta^{3} a_{2}}{24 a_{4}^{2}}\left(4 a_{0}-\Theta L_{x} L_{y} L_{z} \frac{a_{1} a_{3}}{a_{4}}\right) \\
& -\Theta^{2} \frac{a_{0}}{8 a_{4}^{2}}\left(4 \Theta a_{2}-\Theta^{2} \frac{a_{3}^{2}}{a_{4}}\right)-\frac{\Theta^{3} a_{2}^{3}}{54 a_{4}^{3}}-L_{x}^{2} L_{y}^{2} L_{z}^{2} \frac{\Theta^{4} a_{1}^{2}}{8 a_{4}^{2}}, \\
& p=\Theta^{2} \frac{4 a_{0} a_{4}-\Theta L_{x} L_{y} L_{z} a_{1} a_{3}}{12 a_{4}^{2}}-\frac{\Theta a_{2}}{18 a_{4}},
\end{aligned}
$$




$$
\begin{aligned}
& \alpha_{1 \Phi_{I}}=\frac{R_{I 1}}{\Theta L_{x} L_{y} L_{z}}+\frac{S_{I I 20}}{\Theta L_{x} L_{y} L_{z}}+\frac{1}{L_{x} L_{y} L_{z}} \int_{0}^{L_{x}} \int_{0}^{L_{y}} \int_{0}^{L_{z}} f_{\Phi_{I}}(x, y, z) d z d y d x, \\
& \alpha_{1 \Phi_{V}}=\frac{R_{V 1}}{\Theta L_{x} L_{y} L_{z}}+\frac{S_{V V 20}}{\Theta L_{x} L_{y} L_{z}}+\frac{1}{L_{x} L_{y} L_{z}} \int_{0}^{L_{x}} \int_{0}^{L_{y}} \int_{0}^{L_{z}} f_{\Phi_{V}}(x, y, z) d z d y d x,
\end{aligned}
$$

where $R_{\rho i}=\int_{0}^{\Theta}(\Theta-t) \int_{0}^{L_{x}} \int_{0}^{L_{y}} \int_{0}^{L_{z}} k_{I}(x, y, z, T) I_{1}^{i}(x, y, z, t) d z d y d x d t$.

We determine approximations of the second and higher orders of concentrations of dopant and radiation defects framework standard iterative procedure of method of averaging of function corrections [28]. Framework this procedure to determine approximations of the $n$-th order of concentrations of dopant and radiation defects we replace the required concentrations in the Equations (1c), (3c), (5c) on the following sum $\alpha_{n \rho}+\rho_{n-1}(x, y, z, t)$. The replacement leads to the following transformation of the appropriate equations:

$$
\begin{aligned}
\frac{\partial C_{2}(x, y, z, t)}{\partial t}= & \frac{\partial}{\partial x}\left(\left\{1+\xi \frac{\left[\alpha_{2 C}+C_{1}(x, y, z, t)\right]^{\gamma}}{P^{\gamma}(x, y, z, T)}\right\}\right. \\
& \times\left[1+\varsigma_{1} \frac{V(x, y, z, t)}{V^{*}}+\varsigma_{2} \frac{V^{2}(x, y, z, t)}{\left(V^{*}\right)^{2}}\right] \\
& \left.\times D_{L}(x, y, z, T) \frac{\partial C_{1}(x, y, z, t)}{\partial x}\right) \\
& +\frac{\partial}{\partial y}\left(\left[1+\varsigma_{1} \frac{V(x, y, z, t)}{\left.V^{*}+\varsigma_{2} \frac{V^{2}(x, y, z, t)}{\left(V^{*}\right)^{2}}\right] \frac{\partial C_{1}(x, y, z, t)}{\partial y}}\right.\right. \\
& \left.\times D_{L}(x, y, z, T)\left\{1+\xi \frac{\left[\alpha_{2 C}+C_{1}(x, y, z, t)\right]^{\gamma}}{P^{\gamma}(x, y, z, T)}\right\}\right)
\end{aligned}
$$




$$
\begin{aligned}
& +\frac{\partial}{\partial y}\left(\left[1+\varsigma_{1} \frac{V(x, y, z, t)}{V^{*}}+\varsigma_{2} \frac{V^{2}(x, y, z, t)}{\left(V^{*}\right)^{2}}\right]\right. \\
& \left.\times D_{L}(x, y, z, T) \frac{\partial C_{1}(x, y, z, t)}{\partial z}\left\{1+\xi \frac{\left[\alpha_{2 C}+C_{1}(x, y, z, t)\right]^{\gamma}}{P^{\gamma}(x, y, z, T)}\right\}\right) \\
& +f_{C}(x, y, z) \delta(t)+\frac{\partial}{\partial x}\left[\frac{D_{C S}}{\bar{V} k T} \frac{\partial \mu_{2}(x, y, z, t)}{\partial x}\right] \\
& +\frac{\partial}{\partial y}\left[\frac{D_{C S}}{\bar{V} k T} \frac{\partial \mu_{2}(x, y, z, t)}{\partial y}\right]+\frac{\partial}{\partial z}\left[\frac{D_{C S}}{\bar{V} k T} \frac{\partial \mu_{2}(x, y, z, t)}{\partial z}\right] \\
& +\Omega \frac{\partial}{\partial x}\left\{\frac{D_{S}}{k T} \nabla_{S} \mu_{1}(x, y, z, t) \int_{0}^{L_{z}}\left[\alpha_{2 C}+C(x, y, W, t)\right] d W\right\} \\
& +\Omega \frac{\partial}{\partial y}\left\{\frac{D_{S}}{k T} \nabla_{S} \mu_{1}(x, y, z, t) \int_{0}^{L_{z}}\left[\alpha_{2 C}+C(x, y, W, t)\right] d W\right\},
\end{aligned}
$$

$$
\begin{aligned}
\frac{\partial I_{2}(x, y, z, t)}{\partial t}= & \frac{\partial}{\partial x}\left[D_{I}(x, y, z, T) \frac{\partial I_{1}(x, y, z, t)}{\partial x}\right] \\
& +\frac{\partial}{\partial y}\left[D_{I}(x, y, z, T) \frac{\partial I_{1}(x, y, z, t)}{\partial y}\right] \\
& +\frac{\partial}{\partial z}\left[D_{I}(x, y, z, T) \frac{\partial I_{1}(x, y, z, t)}{\partial z}\right] \\
& -k_{I, I}(x, y, z, T)\left[\alpha_{1 I}+I_{1}(x, y, z, t)\right]^{2} \\
& -k_{I, V}(x, y, z, T)\left[\alpha_{1 I}+I_{1}(x, y, z, t)\right]\left[\alpha_{1 V}+V_{1}(x, y, z, t)\right] \\
& +\Omega \frac{\partial}{\partial x}\left\{\nabla_{S} \mu(x, y, z, t) \int_{0}^{L_{z}}\left[\alpha_{2 I}+I_{1}(x, y, W, t)\right] d W \frac{D_{I S}}{k T}\right\}
\end{aligned}
$$




$$
\begin{aligned}
& +\Omega \frac{\partial}{\partial y}\left\{\frac{D_{I S}}{k T} \nabla_{S} \mu(x, y, z, t) \int_{0}^{L_{z}}\left[\alpha_{2 I}+I_{1}(x, y, W, t)\right] d W\right\} \\
& +\frac{\partial}{\partial x} \int_{0}^{t} \frac{\partial \mu_{2}(x, y, z, t)}{\partial x} \frac{D_{I S}}{\bar{V} k T} d \tau+\frac{\partial}{\partial y} \int_{0}^{t} \frac{D_{I S}}{\bar{V} k T} \frac{\partial \mu_{2}(x, y, z, t)}{\partial y} d \tau \\
& +\frac{\partial}{\partial z} \int_{0}^{t} \frac{D_{I S}}{\bar{V} k T} \frac{\partial \mu_{2}(x, y, z, t)}{\partial z} d \tau \\
& \frac{\partial V_{2}(x, y, z, t)}{\partial t}=\frac{\partial}{\partial x}\left[D_{V}(x, y, z, T) \frac{\partial V_{1}(x, y, z, t)}{\partial x}\right] \\
& +\frac{\partial}{\partial y}\left[D_{V}(x, y, z, T) \frac{\partial V_{1}(x, y, z, t)}{\partial y}\right] \\
& +\frac{\partial}{\partial z}\left[D_{V}(x, y, z, T) \frac{\partial V_{1}(x, y, z, t)}{\partial z}\right] \\
& -k_{V, V}(x, y, z, T)\left[\alpha_{1 V}+V_{1}(x, y, z, t)\right]^{2} \\
& -k_{I, V}(x, y, z, T)\left[\alpha_{1 I}+I_{1}(x, y, z, t)\right]\left[\alpha_{1 V}+V_{1}(x, y, z, t)\right] \\
& +\Omega \frac{\partial}{\partial x}\left\{\nabla_{S} \mu(x, y, z, t) \int_{0}^{L_{z}}\left[\alpha_{2 V}+V_{1}(x, y, W, t)\right] d W \frac{D_{V S}}{k T}\right\} \\
& +\Omega \frac{\partial}{\partial y}\left\{\frac{D_{V S}}{k T} \nabla_{S} \mu(x, y, z, t) \int_{0}^{L_{z}}\left[\alpha_{2 V}+V_{1}(x, y, W, t)\right] d W\right\} \\
& +\frac{\partial}{\partial x} \int_{0}^{t} \frac{\partial \mu_{2}(x, y, z, t)}{\partial x} \frac{D_{V S}}{\bar{V} k T} d \tau+\frac{\partial}{\partial y} \int_{0}^{t} \frac{D_{V S}}{\bar{V} k T} \frac{\partial \mu_{2}(x, y, z, t)}{\partial y} d \tau \\
& +\frac{\partial}{\partial z} \int_{0}^{t} \frac{D_{V S}}{\bar{V} k T} \frac{\partial \mu_{2}(x, y, z, t)}{\partial z} d \tau
\end{aligned}
$$




$$
\begin{aligned}
\frac{\partial \Phi_{2 I}(x, y, z, t)}{\partial t}= & \frac{\partial}{\partial x}\left[D_{\Phi_{I}}(x, y, z, T) \frac{\partial \Phi_{1 I}(x, y, z, t)}{\partial x}\right] \\
& +\frac{\partial}{\partial y}\left[D_{\Phi_{I}}(x, y, z, T) \frac{\partial \Phi_{1 I}(x, y, z, t)}{\partial y}\right] \\
& +\Omega \frac{\partial}{\partial x}\left\{\frac{D_{\Phi_{I} S}}{k T} \nabla_{S} \mu(x, y, z, t) \int_{0}^{L_{z}}\left[\alpha_{2 \Phi_{I}}+\Phi_{1 I}(x, y, W, t)\right] d W\right\} \\
& +k_{I, I}(x, y, z, T) I^{2}(x, y, z, t) \\
& +\Omega \frac{\partial}{\partial y}\left\{\frac{D_{\Phi_{I} S}}{k T} \nabla_{S} \mu(x, y, z, t) \int_{0}^{L_{z}}\left[\alpha_{2 \Phi_{I}}+\Phi_{1 I}(x, y, W, t)\right] d W\right\} \\
& +k_{I}(x, y, z, T) I(x, y, z, t)+\frac{\partial}{\partial x}\left[\frac{D_{\Phi_{I} S}}{\bar{V} k T} \frac{\partial \mu_{2}(x, y, z, t)}{\partial x}\right] \\
& +\frac{\partial}{\partial y}\left[\frac{D_{\Phi_{I} S}}{\bar{V} k T} \frac{\partial \mu_{2}(x, y, z, t)}{\partial y}\right]+\frac{\partial}{\partial z}\left[\frac{D_{\Phi_{I} S}}{\bar{V} k T} \frac{\partial \mu_{2}(x, y, z, t)}{\partial z}\right] \\
& +\frac{\partial}{\partial z}\left[D_{\Phi_{I}}(x, y, z, T) \frac{\partial \Phi_{1 I}(x, y, z, t)}{\partial z}\right]+f_{\Phi_{I}}(x, y, z) \delta(t) ;
\end{aligned}
$$$$
\frac{\partial \Phi_{2 V}(x, y, z, t)}{\partial t}=\frac{\partial}{\partial x}\left[D_{\Phi_{V}}(x, y, z, T) \frac{\partial \Phi_{1 V}(x, y, z, t)}{\partial x}\right]
$$$$
+\frac{\partial}{\partial y}\left[D_{\Phi_{V}}(x, y, z, T) \frac{\partial \Phi_{1 V}(x, y, z, t)}{\partial y}\right]
$$$$
+\Omega \frac{\partial}{\partial x}\left\{\frac{D_{\Phi_{V}} S}{k T} \nabla_{S} \mu(x, y, z, t) \int_{0}^{L_{z}}\left[\alpha_{2 \Phi_{V}}+\Phi_{1 V}(x, y, W, t)\right] d W\right\}
$$$$
+k_{V, V}(x, y, z, T) V^{2}(x, y, z, t)
$$ 


$$
\begin{aligned}
& +\Omega \frac{\partial}{\partial y}\left\{\frac{D_{\Phi_{V} S}}{k T} \nabla_{S} \mu(x, y, z, t) \int_{0}^{L_{z}}\left[\alpha_{2 \Phi_{V}}+\Phi_{1 V}(x, y, W, t)\right] d W\right\} \\
& +k_{V}(x, y, z, T) V(x, y, z, t)+\frac{\partial}{\partial x}\left[\frac{D_{\Phi_{V} S}}{\bar{V} k T} \frac{\partial \mu_{2}(x, y, z, t)}{\partial x}\right] \\
& +\frac{\partial}{\partial y}\left[\frac{D_{\Phi_{V} S}}{\bar{V} k T} \frac{\partial \mu_{2}(x, y, z, t)}{\partial y}\right]+\frac{\partial}{\partial z}\left[\frac{D_{\Phi_{V} S}}{\bar{V} k T} \frac{\partial \mu_{2}(x, y, z, t)}{\partial z}\right] \\
& +\frac{\partial}{\partial z}\left[D_{\Phi_{V}}(x, y, z, T) \frac{\partial \Phi_{1 V}(x, y, z, t)}{\partial z}\right]+f_{\Phi_{V}}(x, y, z) \delta(t) .
\end{aligned}
$$

Integration of the left and the right sides of Equations (1d), (3d) and (5d) gives us possibility to obtain relations for the required concentrations in the final form

$$
\begin{aligned}
C_{2}(x, y, z, t)= & \frac{\partial}{\partial x} \int_{0}^{t}\left\{1+\xi \frac{\left[\alpha_{2 C}+C_{1}(x, y, z, \tau)\right]^{\gamma}}{P^{\gamma}(x, y, z, T)}\right\} \\
& \times\left[1+\varsigma_{1} \frac{V(x, y, z, \tau)}{V^{*}}+\varsigma_{2} \frac{V^{2}(x, y, z, \tau)}{\left(V^{*}\right)^{2}}\right] \\
& \times D_{L}(x, y, z, T) \frac{\partial C_{1}(x, y, z, \tau)}{\partial x} d \tau \\
& +\frac{\partial}{\partial y} \int_{0}^{t} D_{L}(x, y, z, T)\left[1+\varsigma_{1} \frac{V(x, y, z, \tau)}{V^{*}}+\varsigma_{2} \frac{V^{2}(x, y, z, \tau)}{\left(V^{*}\right)^{2}}\right] \\
& \times \frac{\partial C_{1}(x, y, z, \tau)}{\partial y}\left\{1+\xi \frac{\left[\alpha_{2 C}+C_{1}(x, y, z, t)\right]^{\gamma}}{P^{\gamma}(x, y, z, T)}\right\} \\
& +\frac{\partial}{\partial z} \int_{0}^{t}\left[1+\varsigma_{1} \frac{V(x, y, z, \tau)}{\left.V^{*}+\varsigma_{2} \frac{V^{2}(x, y, z, \tau)}{\left(V^{*}\right)^{2}}\right]}\right.
\end{aligned}
$$




$$
\begin{aligned}
& \times D_{L}(x, y, z, T) \frac{\partial C_{1}(x, y, z, \tau)}{\partial z}\left\{1+\xi \frac{\left[\alpha_{2 C}+C_{1}(x, y, z, \tau)\right]^{\gamma}}{P^{\gamma}(x, y, z, T)}\right\} d \tau \\
& +f_{C}(x, y, z)+\Omega \frac{\partial}{\partial x} \int_{0}^{t} \frac{D_{S}}{k T} \nabla_{S} \mu(x, y, z, \tau) \int_{0}^{L_{z}}\left[\alpha_{2 C}\right. \\
& \left.+C_{1}(x, y, W, \tau)\right] d W d \tau+\frac{\partial}{\partial y} \int_{0}^{t} \nabla_{S} \mu(x, y, z, \tau) \\
& \times \Omega \frac{D_{S}}{k T} \int_{0}^{L_{z}}\left[\alpha_{2 C}+C_{1}(x, y, W, \tau)\right] d W d \tau \\
& +\frac{\partial}{\partial x}\left[\frac{D_{C S}}{\bar{V} k T} \frac{\partial \mu_{2}(x, y, z, t)}{\partial x}\right]+\frac{\partial}{\partial y}\left[\frac{D_{C S}}{\bar{V} k T} \frac{\partial \mu_{2}(x, y, z, t)}{\partial y}\right] \\
& +\int_{0}^{t} k_{I, V}(x, y, z, T)\left[\alpha_{2 I}+I_{1}(x, y, z, \tau)\right]\left[\alpha_{2 V}+V_{1}(x, y, z, \tau)\right] d \tau \\
& +\frac{\partial}{\partial z}\left[\frac{D_{C S}}{\bar{V} k T} \frac{\partial \mu_{2}(x, y, z, t)}{\partial z}\right], \\
& +\frac{\partial}{\partial y} \int_{0}^{t} \int_{0}^{t} \int_{I} D_{I}(x, y, z, T) \frac{\partial I_{1}(x, y, z, \tau)}{\partial y} d \tau \\
I_{2}(x, y, z, t)= & \\
& \\
& \\
& \\
& \\
& \\
& \\
&
\end{aligned}
$$




$$
\begin{aligned}
& +\frac{\partial}{\partial x} \int_{0}^{t} \nabla_{S} \mu(x, y, z, \tau) \Omega \frac{D_{I S}}{k T} \int_{0}^{L_{z}}\left[\alpha_{2 I}+I_{1}(x, y, W, \tau)\right] d W d \tau \\
& +\frac{\partial}{\partial y} \int_{0}^{t} \nabla_{S} \mu(x, y, z, \tau) \int_{0}^{L_{z}}\left[\alpha_{2 I}+I_{1}(x, y, W, \tau)\right] \Omega \frac{D_{I S}}{k T} d W d \tau \\
& +\frac{\partial}{\partial x}\left[\frac{D_{I S}}{\bar{V} k T} \frac{\partial \mu_{2}(x, y, z, t)}{\partial x}\right]+\frac{\partial}{\partial y}\left[\frac{D_{I S}}{\bar{V} k T} \frac{\partial \mu_{2}(x, y, z, t)}{\partial y}\right] \\
& +\frac{\partial}{\partial z}\left[\frac{D_{I S}}{\bar{V} k T} \frac{\partial \mu_{2}(x, y, z, t)}{\partial z}\right]+f_{I}(x, y, z) \\
& V_{2}(x, y, z, t)=\frac{\partial}{\partial x} \int_{0}^{t} D_{V}(x, y, z, T) \frac{\partial V_{1}(x, y, z, \tau)}{\partial x} d \tau \\
& +\frac{\partial}{\partial y} \int_{0}^{t} D_{V}(x, y, z, T) \frac{\partial V_{1}(x, y, z, \tau)}{\partial y} d \tau \\
& +\frac{\partial}{\partial z} \int_{0}^{t} D_{V}(x, y, z, T) \frac{\partial V_{1}(x, y, z, \tau)}{\partial z} d \tau \\
& -\int_{0}^{t} k_{V, V}(x, y, z, T)\left[\alpha_{2 V}+V_{1}(x, y, z, \tau)\right]^{2} d \tau \\
& -\int_{0}^{t} k_{I, V}(x, y, z, T)\left[\alpha_{2 I}+I_{1}(x, y, z, \tau)\right]\left[\alpha_{2 V}+V_{1}(x, y, z, \tau)\right] d \tau \\
& +\frac{\partial}{\partial x} \int_{0}^{t} \nabla_{S} \mu(x, y, z, \tau) \Omega \frac{D_{V S}}{k T} \int_{0}^{L_{z}}\left[\alpha_{2 V}+V_{1}(x, y, W, \tau)\right] d W d \tau \\
& +\frac{\partial}{\partial y} \int_{0}^{t} \nabla_{S} \mu(x, y, z, \tau) \int_{0}^{L_{z}}\left[\alpha_{2 V}+V_{1}(x, y, W, \tau)\right] \Omega \frac{D_{V S}}{k T} d W d \tau
\end{aligned}
$$




$$
\begin{aligned}
& +\frac{\partial}{\partial x}\left[\frac{D_{V S}}{\bar{V} k T} \frac{\partial \mu_{2}(x, y, z, t)}{\partial x}\right]+\frac{\partial}{\partial y}\left[\frac{D_{V S}}{\bar{V} k T} \frac{\partial \mu_{2}(x, y, z, t)}{\partial y}\right] \\
& +\frac{\partial}{\partial z}\left[\frac{D_{V S}}{\bar{V} k T} \frac{\partial \mu_{2}(x, y, z, t)}{\partial z}\right]+f_{V}(x, y, z), \\
& \Phi_{2 I}(x, y, z, t)=\frac{\partial}{\partial x} \int_{0}^{t} D_{\Phi_{I}}(x, y, z, T) \frac{\partial \Phi_{1 I}(x, y, z, \tau)}{\partial x} d \tau \\
& +\frac{\partial}{\partial y} \int_{0}^{t} \frac{\partial \Phi_{1 I}(x, y, z, \tau)}{\partial y} D_{\Phi_{I}}(x, y, z, T) d \tau \\
& +\frac{\partial}{\partial z} \int_{0}^{t} D_{\Phi_{I}}(x, y, z, T) \frac{\partial \Phi_{1 I}(x, y, z, \tau)}{\partial z} d \tau \\
& +\Omega \frac{\partial}{\partial x} \int_{0}^{t} \nabla_{S} \mu(x, y, z, \tau) \frac{D_{\Phi_{I}} S}{k T} \int_{0}^{L_{z}}\left[\alpha_{2 \Phi_{I}}+\Phi_{1 I}(x, y, W, \tau)\right] d W d \tau \\
& +\Omega \frac{\partial}{\partial y} \int_{0}^{t} \frac{D_{\Phi_{I}} S}{k T} \int_{0}^{L_{z}}\left[\alpha_{2 \Phi_{I}}+\Phi_{1 I}(x, y, W, \tau)\right] d W \nabla_{S} \mu(x, y, z, \tau) d \tau \\
& +\int_{0}^{t} k_{I, I}(x, y, z, T) I^{2}(x, y, z, \tau) d \tau+\frac{\partial}{\partial x} \int_{0}^{t} \frac{D_{\Phi_{I} S}}{\bar{V} k T} \frac{\partial \mu_{2}(x, y, z, \tau)}{\partial x} d \tau \\
& +\frac{\partial}{\partial y} \int_{0}^{t} \frac{D_{\Phi_{I} S}}{\bar{V} k T} \frac{\partial \mu_{2}(x, y, z, \tau)}{\partial y} d \tau \\
& +\frac{\partial}{\partial z} \int_{0}^{t} \frac{D_{\Phi_{I} S}}{\bar{V} k T} \frac{\partial \mu_{2}(x, y, z, \tau)}{\partial z} d \tau \\
& +f_{\Phi_{I}}(x, y, z)+\int_{0}^{t} k_{I}(x, y, z, T) I(x, y, z, \tau) d \tau
\end{aligned}
$$




$$
\begin{aligned}
& \Phi_{2 V}(x, y, z, t)=\frac{\partial}{\partial x} \int_{0}^{t} D_{\Phi_{V}}(x, y, z, T) \frac{\partial \Phi_{1 V}(x, y, z, \tau)}{\partial x} d \tau \\
& +\frac{\partial}{\partial y} \int_{0}^{t} \frac{\partial \Phi_{1 V}(x, y, z, \tau)}{\partial y} \times D_{\Phi_{V}}(x, y, z, T) d \tau \\
& +\frac{\partial}{\partial z} \int_{0}^{t} D_{\Phi_{V}}(x, y, z, T) \frac{\partial \Phi_{1 V}(x, y, z, \tau)}{\partial z} d \tau \\
& +\Omega \frac{\partial}{\partial x} \int_{0}^{t} \nabla_{S} \mu(x, y, z, \tau) \\
& \times \frac{D_{\Phi_{V}} S}{k T} \int_{0}^{L_{z}}\left[\alpha_{2 \Phi_{V}}+\Phi_{1 V}(x, y, W, \tau)\right] d W d \tau \\
& +\Omega \frac{\partial}{\partial y} \int_{0}^{t} \frac{D_{\Phi_{V}} S}{k T} \int_{0}^{L_{z}}\left[\alpha_{2 \Phi_{V}}+\Phi_{1 V}(x, y, W, \tau)\right] d W \\
& \times \nabla_{S} \mu(x, y, z, \tau) d \tau+\int_{0}^{t} k_{V, V}(x, y, z, T) V^{2}(x, y, z, \tau) d \tau \\
& +\frac{\partial}{\partial x} \int_{0}^{t} \frac{D_{\Phi_{V} S}}{\bar{V} k T} \frac{\partial \mu_{2}(x, y, z, \tau)}{\partial x} d \tau \\
& +\frac{\partial}{\partial y} \int_{0}^{t} \frac{D_{\Phi_{V} S}}{\bar{V} k T} \frac{\partial \mu_{2}(x, y, z, \tau)}{\partial y} d \tau \\
& +\frac{\partial}{\partial z} \int_{0}^{t} \frac{D_{\Phi_{V} S}}{\bar{V} k T} \frac{\partial \mu_{2}(x, y, z, \tau)}{\partial z} d \tau+f_{\Phi_{V}}(x, y, z) \\
& +\int_{0}^{t} k_{V}(x, y, z, T) V(x, y, z, \tau) d \tau
\end{aligned}
$$


Average values of the second-order approximations of required approximations by using the following standard relation [28]:

$$
\alpha_{2 \rho}=\frac{1}{\Theta L_{x} L_{y} L_{z}} \int_{0}^{\Theta} \int_{0}^{L_{x}} \int_{0}^{L_{y}} \int_{0}^{L_{z}}\left[\rho_{2}(x, y, z, t)-\rho_{1}(x, y, z, t)\right] d z d y d x d t .
$$

Substitution of the relations (1e), (3e), (5e) into relation (10) gives us possibility to obtain relations for required average values $\alpha_{2 \rho}$

$$
\begin{aligned}
& \alpha_{2 C}=0, \alpha_{2 \Phi I}=0, \alpha_{2 \Phi V}=0, \\
& \alpha_{2 V}=\sqrt{\frac{\left(b_{3}+E\right)^{2}}{4 b_{4}^{2}}-4\left(F+\frac{\Theta a_{3} F+\Theta^{2} L_{x} L_{y} L_{z} b_{1}}{b_{4}}\right)}-\frac{b_{3}+E}{4 b_{4}}, \\
& \alpha_{2 I}=\frac{C_{V}-\alpha_{2 V}^{2} S_{V V 00}-\alpha_{2 V}\left(2 S_{V V 01}+S_{I V 10}+\Theta L_{x} L_{y} L_{z}\right)-S_{V V 02}-S_{I V 11}}{S_{I V 01}+\alpha_{2 V} S_{I V 00}},
\end{aligned}
$$

where

$$
\begin{aligned}
b_{4}= & \frac{1}{\Theta L_{x} L_{y} L_{z}} S_{I V 00}^{2} S_{V V 00}-\frac{1}{\Theta L_{x} L_{y} L_{z}} S_{V V 00}^{2} S_{I I 00}, \\
b_{3}= & -\frac{S_{I I 00} S_{V V 00}}{\Theta L_{x} L_{y} L_{z}}\left(2 S_{V V 01}+S_{I V 10}+\Theta L_{x} L_{y} L_{z}\right) \\
& +\frac{S_{I V 00} S_{V V 00}}{\Theta L_{x} L_{y} L_{z}}\left(S_{I V 01}+2 S_{I I 10}+S_{I V 01}+\Theta L_{x} L_{y} L_{z}\right) \\
& +\frac{S_{I V 00}^{2}}{\Theta L_{x} L_{y} L_{z}}\left(2 S_{V V 01}+S_{I V 10}+\Theta L_{x} L_{y} L_{z}\right)-\frac{S_{I V 00}^{2} S_{I V 10}}{\Theta L_{x}^{3} L_{y}^{3} L_{z}^{3}}, \\
b_{2}= & \frac{S_{I I 00} S_{V V 00}}{\Theta L_{x} L_{y} L_{z}}\left(S_{V V 02}+S_{I V 11}+C_{V}\right)-\left(S_{I V 10}-2 S_{V V 01}+\Theta L_{x} L_{y} L_{z}\right)^{2} \\
& +\frac{S_{I V 01} S_{V V 00}}{\Theta L_{x} L_{y} L_{z}}\left(\Theta L_{x} L_{y} L_{z}+2 S_{I I 10}+S_{I V 01}\right)
\end{aligned}
$$




$$
\begin{aligned}
& +\frac{S_{I V 00}}{\Theta L_{x} L_{y} L_{z}}\left(S_{I V 01}+2 S_{I I 10}+2 S_{I V 01}+\Theta L_{x} L_{y} L_{z}\right)\left(2 S_{V V 01}\right. \\
& \left.+\Theta L_{x} L_{y} L_{z}+S_{I V 10}\right)-\frac{S_{I V 00}^{2}}{\Theta L_{x} L_{y} L_{z}}\left(C_{V}-S_{V V 02}-S_{I V 11}\right) \\
& +\frac{C_{I} S_{I V 00}^{2}}{\Theta^{2} L_{x}^{2} L_{y}^{2} L_{z}^{2}}-\frac{2 S_{I V 10}}{\Theta L_{x} L_{y} L_{z}} S_{I V 00} S_{I V 01}, \\
& b_{1}=S_{I I 00} \frac{S_{I V 11}+S_{V V 02}+C_{V}}{\Theta L_{x} L_{y} L_{z}}\left(2 S_{V V 01}+S_{I V 10}+\Theta L_{x} L_{y} L_{z}\right) \\
& +\frac{S_{I V 01}}{\Theta L_{x} L_{y} L_{z}}\left(\Theta L_{x} L_{y} L_{z}+2 S_{I I 10}+S_{I V 01}\right) \\
& \times\left(2 S_{V V 01}+S_{I V 10}+\Theta L_{x} L_{y} L_{z}\right)-\frac{S_{I V 10} S_{I V 01}^{2}}{\Theta L_{x} L_{y} L_{z}} \\
& -\frac{S_{I V 00}}{\Theta L_{x} L_{y} L_{z}}\left(3 S_{I V 01}+2 S_{I I 10}+\Theta L_{x} L_{y} L_{z}\right) \\
& \times\left(C_{V}-S_{V V 02}-S_{I V 11}\right)+2 C_{I} S_{I V 00} S_{I V 01}, \\
& b_{0}=\frac{S_{I I 00}}{\Theta L_{x} L_{y} L_{z}}\left(S_{I V 00}+S_{V V 02}\right)^{2} \\
& -\frac{S_{I V 01}}{L_{x} L_{y} L_{z}} \frac{1}{\Theta}\left(\Theta L_{x} L_{y} L_{z}+2 S_{I I 10}+S_{I V 01}\right) \\
& \times\left(C_{V}-S_{V V 02}-S_{I V 11}\right)+2 C_{I} S_{I V 01}^{2} \\
& -S_{I V 01} \frac{C_{V}-S_{V V 02}-S_{I V 11}}{\Theta L_{x} L_{y} L_{z}}\left(\Theta L_{x} L_{y} L_{z}+2 S_{I I 10}+S_{I V 01}\right), \\
& C_{I}=\frac{\alpha_{1 I} \alpha_{1 V}}{\Theta L_{x} L_{y} L_{z}} S_{I V 00}+\frac{\alpha_{1 I}^{2} S_{I I 00}}{\Theta L_{x} L_{y} L_{z}}-\frac{S_{I I 20} S_{I I 20}}{\Theta L_{x} L_{y} L_{z}}-\frac{S_{I V 11}}{\Theta L_{x} L_{y} L_{z}}, \\
& C_{V}=\alpha_{1 I} \alpha_{1 V} S_{I V 00}+\alpha_{1 V}^{2} S_{V V 00}-S_{V V 02}-S_{I V 11},
\end{aligned}
$$




$$
\begin{aligned}
E= & \sqrt{8 y+\Theta^{2} \frac{a_{3}^{2}}{a_{4}^{2}}-4 \Theta \frac{a_{2}}{a_{4}}}, \\
F= & \frac{\Theta a_{2}}{6 a_{4}}+\sqrt[3]{\sqrt{r^{2}+s^{3}}-r}-\sqrt[3]{\sqrt{r^{2}+s^{3}}+r}, \\
r= & \frac{\Theta^{3} b_{2}}{24 b_{4}^{2}}\left(4 b_{0}-\Theta L_{x} L_{y} L_{z} \frac{b_{1} b_{3}}{b_{4}}\right)-\frac{\Theta^{3} b_{2}^{3}}{54 b_{4}^{3}} \\
& -b_{0} \frac{\Theta^{2}}{8 b_{4}^{2}}\left(4 \Theta b_{2}-\Theta^{2} \frac{b_{3}^{2}}{b_{4}}\right)-L_{x}^{2} L_{y}^{2} L_{z}^{2} \frac{\Theta^{4} b_{1}^{2}}{8 b_{4}^{2}}, \\
s= & \Theta^{2} \frac{4 b_{0} b_{4}-\Theta L_{x} L_{y} L_{z} b_{1} b_{3}}{12 b_{4}^{2}}-\frac{\Theta b_{2}}{18 b_{4}} .
\end{aligned}
$$

Farther we determine solutions of Equations (8), i.e., components of displacement vector. To determine the first-order approximations of the considered components framework method of averaging of function corrections we replace the required functions in the right sides of the equations by their not yet known average values $\alpha_{i}$. The substitution leads to the following result:

$$
\begin{gathered}
\rho(z) \frac{\partial^{2} u_{1 x}(x, y, z, t)}{\partial t^{2}}=-K(z) \beta(z) \frac{\partial T(x, y, z, t)}{\partial x}, \\
\rho(z) \frac{\partial^{2} u_{1 y}(x, y, z, t)}{\partial t^{2}}=-K(z) \beta(z) \frac{\partial T(x, y, z, t)}{\partial y}, \\
\rho(z) \frac{\partial^{2} u_{1 z}(x, y, z, t)}{\partial t^{2}}=-K(z) \beta(z) \frac{\partial T(x, y, z, t)}{\partial z} .
\end{gathered}
$$

Integration of the left and the right sides of the above relations on time $t$ leads to the following result:

$$
\begin{aligned}
u_{1 x}(x, y, z, t)= & u_{0 x}+K(z) \frac{\beta(z)}{\rho(z)} \frac{\partial}{\partial x} \int_{0}^{t} \int_{0}^{\vartheta} T(x, y, z, \tau) d \tau d \vartheta \\
& -K(z) \frac{\beta(z)}{\rho(z)} \frac{\partial}{\partial x} \int_{0}^{\infty} \int_{0}^{\vartheta} T(x, y, z, \tau) d \tau d \vartheta
\end{aligned}
$$




$$
\begin{aligned}
u_{1 y}(x, y, z, t)= & u_{0 y}+K(z) \frac{\beta(z)}{\rho(z)} \frac{\partial}{\partial y} \int_{0}^{t} \int_{0}^{\vartheta} T(x, y, z, \tau) d \tau d \vartheta \\
& -K(z) \frac{\beta(z)}{\rho(z)} \frac{\partial}{\partial y} \int_{0}^{\infty} \int_{0}^{\vartheta} T(x, y, z, \tau) d \tau d \vartheta \\
u_{1 z}(x, y, z, t)= & u_{0 z}+K(z) \frac{\beta(z)}{\rho(z)} \frac{\partial}{\partial z} \int_{0}^{t} \int_{0}^{\vartheta} T(x, y, z, \tau) d \tau d \vartheta \\
& -K(z) \frac{\beta(z)}{\rho(z)} \frac{\partial}{\partial z} \int_{0}^{\infty} \int_{0}^{\vartheta} T(x, y, z, \tau) d \tau d \vartheta .
\end{aligned}
$$

Approximations of the second and higher orders of components of displacement vector could be determined by using standard replacement of the required components on the following sums $\alpha_{i}+u_{i}(x, y, z, t)$ [28].

The replacement leads to the following result:

$$
\begin{aligned}
\rho(z) \frac{\partial^{2} u_{2 x}(x, y, z, t)}{\partial t^{2}}= & \left\{K(z)+\frac{5 E(z)}{6[1+\sigma(z)]}\right\} \frac{\partial^{2} u_{1 x}(x, y, z, t)}{\partial x^{2}} \\
& +\left\{K(z)-\frac{E(z)}{3[1+\sigma(z)]}\right\} \frac{\partial^{2} u_{1 y}(x, y, z, t)}{\partial x \partial y} \\
& +\frac{E(z)}{2[1+\sigma(z)]}\left[\frac{\partial^{2} u_{1 y}(x, y, z, t)}{\partial y^{2}}+\frac{\partial^{2} u_{1 z}(x, y, z, t)}{\partial z^{2}}\right] \\
& -\frac{\partial T(x, y, z, t)}{\partial x} K(z) \beta(z) \\
& +\left\{K(z)+\frac{E(z)}{3[1+\sigma(z)]}\right\} \frac{\partial^{2} u_{1 z}(x, y, z, t)}{\partial x \partial z},
\end{aligned}
$$




$$
\begin{aligned}
& \rho(z) \frac{\partial^{2} u_{2 y}(x, y, z, t)}{\partial t^{2}}=\frac{E(z)}{2[1+\sigma(z)]}\left[\frac{\partial^{2} u_{1 y}(x, y, z, t)}{\partial x^{2}}+\frac{\partial^{2} u_{1 x}(x, y, z, t)}{\partial x \partial y}\right] \\
& -\frac{\partial T(x, y, z, t)}{\partial y} K(z) \beta(z) \\
& +\frac{\partial}{\partial z}\left\{\frac{E(z)}{2[1+\sigma(z)]}\left[\frac{\partial u_{1 y}(x, y, z, t)}{\partial z}+\frac{\partial u_{1 z}(x, y, z, t)}{\partial y}\right]\right\} \\
& +\frac{\partial^{2} u_{1 y}(x, y, z, t)}{\partial y^{2}}\left\{\frac{5 E(z)}{12[1+\sigma(z)]}+K(z)\right\} \\
& +\left\{K(z)-\frac{E(z)}{6[1+\sigma(z)]}\right\} \frac{\partial^{2} u_{1 y}(x, y, z, t)}{\partial y \partial z} \\
& +K(z) \frac{\partial^{2} u_{1 y}(x, y, z, t)}{\partial x \partial y} \\
& \rho(z) \frac{\partial^{2} u_{2 z}(x, y, z, t)}{\partial t^{2}}=\frac{E(z)}{2[1+\sigma(z)]}\left[\frac{\partial^{2} u_{1 z}(x, y, z, t)}{\partial x^{2}}+\frac{\partial^{2} u_{1 z}(x, y, z, t)}{\partial y^{2}}\right. \\
& \left.+\frac{\partial^{2} u_{1 x}(x, y, z, t)}{\partial x \partial z}+\frac{\partial^{2} u_{1 y}(x, y, z, t)}{\partial y \partial z}\right] \\
& +\frac{\partial}{\partial z}\left\{K ( z ) \left[\frac{\partial u_{1 x}(x, y, z, t)}{\partial x}+\frac{\partial u_{1 y}(x, y, z, t)}{\partial y}\right.\right. \\
& \left.\left.+\frac{\partial u_{1 x}(x, y, z, t)}{\partial z}\right]\right\}+\frac{E(z)}{6[1+\sigma(z)]} \frac{\partial}{\partial z}\left[6 \frac{\partial u_{1 z}(x, y, z, t)}{\partial z}\right. \\
& \left.-\frac{\partial u_{1 x}(x, y, z, t)}{\partial x}-\frac{\partial u_{1 y}(x, y, z, t)}{\partial y}-\frac{\partial u_{1 z}(x, y, z, t)}{\partial z}\right] \\
& \left.\left.-\frac{\partial u_{1 x}(x, y, z, t)}{\partial x}-\frac{\partial u_{1 y}(x, y, z, t)}{\partial y}-\frac{\partial u_{1 z}(x, y, z, t)}{\partial z}\right]\right\} \\
& \times \frac{E(z)}{1+\sigma(z)}-K(z) \beta(z) \frac{\partial T(x, y, z, t)}{\partial z} .
\end{aligned}
$$


Integration of the left and right sides of the above relations on time $t$ leads to the following result

$$
\begin{aligned}
& u_{2 x}(x, y, z, t)=\frac{1}{\rho(z)}\left\{K(z)+\frac{5 E(z)}{6[1+\sigma(z)]}\right\} \frac{\partial^{2}}{\partial x^{2}} \int_{0}^{t} \int_{0}^{\vartheta} u_{1 x}(x, y, z, \tau) d \tau d \vartheta \\
& +\frac{1}{\rho(z)}\left\{K(z)-\frac{E(z)}{3[1+\sigma(z)]}\right\} \frac{\partial^{2}}{\partial x \partial y} \int_{0}^{t} \int_{0}^{\vartheta} u_{1 y}(x, y, z, \tau) d \tau d \vartheta \\
& +\frac{E(z)}{2 \rho(z)}\left[\frac{\partial^{2}}{\partial y^{2}} \int_{0}^{t} \int_{0}^{\vartheta} u_{1 y}(x, y, z, \tau) d \tau d \vartheta\right. \\
& \left.+\frac{\partial^{2}}{\partial z^{2}} \int_{0}^{t} \int_{0}^{\vartheta} u_{1 z}(x, y, z, \tau) d \tau d \vartheta\right] \frac{1}{1+\sigma(z)}+\frac{1}{\rho(z)} \frac{\partial^{2}}{\partial x \partial z} \\
& \times \int_{0}^{t} \int_{0}^{\vartheta} u_{1 z}(x, y, z, \tau) d \tau d \vartheta\left\{K(z)+\frac{E(z)}{3[1+\sigma(z)]}\right\} \\
& -K(z) \frac{\beta(z)}{\rho(z)} \frac{\partial}{\partial x} \int_{0}^{t} \int_{0}^{\vartheta} T(x, y, z, \tau) d \tau d \vartheta \\
& -\frac{\partial^{2}}{\partial x^{2}} \int_{0}^{\infty} \int_{0}^{\vartheta} u_{1 x}(x, y, z, \tau) d \tau d \vartheta \frac{1}{\rho(z)}\left\{K(z)+\frac{5 E(z)}{6[1+\sigma(z)]}\right\} \\
& -\left\{K(z)-\frac{E(z)}{3[1+\sigma(z)]}\right\} \frac{\partial^{2}}{\partial x \partial y} \int_{0}^{\infty} \int_{0}^{\vartheta} u_{1 y}(x, y, z, \tau) d \tau d \vartheta \frac{1}{\rho(z)} \\
& -\frac{E(z)}{2 \rho(z)[1+\sigma(z)]}\left[\frac{\partial^{2}}{\partial y^{2}} \int_{0}^{\infty} \int_{0}^{\vartheta} u_{1 y}(x, y, z, \tau) d \tau d \vartheta\right. \\
& \left.+\frac{\partial^{2}}{\partial z^{2}} \int_{0}^{\infty} \int_{0}^{\vartheta} u_{1 z}(x, y, z, \tau) d \tau d \vartheta\right]
\end{aligned}
$$




$$
\begin{aligned}
& -\frac{1}{\rho(z)}\left\{K(z)+\frac{E(z)}{3[1+\sigma(z)]}\right\} \frac{\partial^{2}}{\partial x \partial z} \int_{0}^{\infty} \int_{0}^{\vartheta} u_{1 z}(x, y, z, \tau) d \tau d \vartheta \\
& +u_{0 x}+K(z) \frac{\beta(z)}{\rho(z)} \frac{\partial}{\partial x} \int_{0}^{\infty} \int_{0}^{\vartheta} T(x, y, z, \tau) d \tau d \vartheta \\
& u_{2 y}(x, y, z, t)=\frac{E(z)}{2 \rho(z)}\left[\frac{\partial^{2}}{\partial x^{2}} \int_{0}^{t} \int_{0}^{\vartheta} u_{1 x}(x, y, z, \tau) d \tau d \vartheta\right. \\
& \left.+\frac{\partial^{2}}{\partial x \partial y} \int_{0}^{t} \int_{0}^{\vartheta} u_{1 x}(x, y, z, \tau) d \tau d \vartheta\right] \frac{1}{1+\sigma(z)} \\
& +\frac{K(z)}{\rho(z)} \frac{\partial^{2}}{\partial x \partial y} \int_{0}^{t} \int_{0}^{\vartheta} u_{1 y}(x, y, z, \tau) d \tau d \vartheta \\
& +\frac{1}{\rho(z)}\left\{\frac{5 E(z)}{12[1+\sigma(z)]}+K(z)\right\} \frac{\partial^{2}}{\partial y^{2}} \int_{0}^{t} \int_{0}^{\vartheta} u_{1 x}(x, y, z, \tau) d \tau d \vartheta \\
& +\frac{1}{2 \rho(z)} \frac{\partial}{\partial z}\left\{\frac { E ( z ) } { 1 + \sigma ( z ) } \left[\frac{\partial}{\partial z} \int_{0}^{t} \int_{0}^{\vartheta} u_{1 y}(x, y, z, \tau) d \tau d \vartheta\right.\right. \\
& \left.\left.+\frac{\partial}{\partial y} \int_{0}^{t} \int_{0}^{\vartheta} u_{1 z}(x, y, z, \tau) d \tau d \vartheta\right]\right\}-K(z) \frac{\beta(z)}{\rho(z)} \int_{0}^{t} \int_{0}^{\vartheta} T(x, y, z, \tau) d \tau d \vartheta \\
& -\left\{\frac{E(z)}{6[1+\sigma(z)]}-K(z)\right\} \frac{1}{\rho(z)} \frac{\partial^{2}}{\partial y \partial z} \int_{0}^{t} \int_{0}^{\vartheta} u_{1 y}(x, y, z, \tau) d \tau d \vartheta \\
& -\frac{E(z)}{2 \rho(z)}\left[\frac{\partial^{2}}{\partial x^{2}} \int_{0}^{\infty} \int_{0}^{\vartheta} u_{1 x}(x, y, z, \tau) d \tau d \vartheta\right.
\end{aligned}
$$




$$
\begin{aligned}
& \left.+\frac{\partial^{2}}{\partial x \partial y} \int_{0}^{\infty} \int_{0}^{\vartheta} u_{1 x}(x, y, z, \tau) d \tau d \vartheta\right] \frac{1}{1+\sigma(z)}-K(z) \frac{\beta(z)}{\rho(z)} \\
& \times \int_{0}^{\infty} \int_{0}^{\vartheta} T(x, y, z, \tau) d \tau d \vartheta-\frac{K(z)}{\rho(z)} \frac{\partial^{2}}{\partial x \partial y} \int_{0}^{\infty} \int_{0}^{\vartheta} u_{1 y}(x, y, z, \tau) d \tau d \vartheta \\
& -\frac{1}{\rho(z)} \frac{\partial^{2}}{\partial y^{2}} \int_{0}^{\infty} \int_{0}^{\vartheta} u_{1 x}(x, y, z, \tau) d \tau d \vartheta\left\{\frac{5 E(z)}{12[1+\sigma(z)]}+K(z)\right\} \\
& \left.\left.+\frac{\partial}{\partial y} \int_{0}^{\infty} \int_{0}^{\vartheta} u_{1 z}(x, y, z, \tau) d \tau d \vartheta\right]\right\} \frac{1}{2 \rho(z)} \\
& -\frac{\partial}{\partial z}\left\{\frac { E ( z ) } { 1 + \sigma ( z ) } \left[\frac{\partial}{\partial z} \int_{0}^{\infty} \int_{0}^{\vartheta} u_{1 y}(x, y, z, \tau) d \tau d \vartheta\right.\right. \\
& -\frac{1}{\rho(z)}\left\{K(z)-\frac{E(z)}{6[1+\sigma(z)]}\right\} \frac{\partial^{2}}{\partial y \partial z} \int_{0}^{\infty} \int_{0}^{\vartheta} u_{1 y}(x, y, z, \tau) d \tau d \vartheta+u_{0 y} \\
& u_{z}(x, y, z, t)=\frac{E(z)}{2[1+\sigma(z)]}\left[\frac{\partial^{2}}{\partial x^{2}} \int_{0}^{\infty} \int_{0}^{\vartheta} u_{1 z}(x, y, z, \tau) d \tau d \vartheta\right. \\
& +\frac{\partial^{2}}{\partial y^{2}} \int_{0}^{\infty} \int_{0}^{\vartheta} u_{1 z}(x, y, z, \tau) d \tau d \vartheta+\frac{\partial^{2}}{\partial x \partial z} \int_{0}^{\infty} \int_{0}^{\vartheta} u_{1 x}(x, y, z, \tau) d \tau d \vartheta \\
& \left.+\frac{\partial^{2}}{\partial y \partial z} \int_{0}^{\infty} \int_{0}^{\vartheta} u_{1 y}(x, y, z, \tau) d \tau d \vartheta\right] \frac{1}{\rho(z)} \\
& +\frac{1}{\rho(z)} \frac{\partial}{\partial z}\left\{K ( z ) \left[\frac{\partial}{\partial x} \int_{0}^{\infty} \int_{0}^{\vartheta} u_{1 x}(x, y, z, \tau) d \tau d \vartheta\right.\right. \\
& \left.\left.+\frac{\partial}{\partial y} \int_{0}^{\infty} \int_{0}^{\vartheta} u_{1 x}(x, y, z, \tau) d \tau d \vartheta+\frac{\partial}{\partial z} \int_{0}^{\infty} \int_{0}^{\vartheta} u_{1 x}(x, y, z, \tau) d \tau d \vartheta\right]\right\}
\end{aligned}
$$




$$
\begin{aligned}
& +\frac{1}{6 \rho(z)} \frac{\partial}{\partial z}\left\{\frac { E ( z ) } { 1 + \sigma ( z ) } \left[6 \frac{\partial}{\partial z} \int_{0}^{\infty} \int_{0}^{\vartheta} u_{1 z}(x, y, z, \tau) d \tau d \vartheta\right.\right. \\
& -\frac{\partial}{\partial x} \int_{0}^{\infty} \int_{0}^{\vartheta} u_{1 x}(x, y, z, \tau) d \tau d \vartheta-\frac{\partial}{\partial y} \int_{0}^{\infty} \int_{0}^{\vartheta} u_{1 y}(x, y, z, \tau) d \tau d \vartheta \\
& \left.\left.-\frac{\partial}{\partial z} \int_{0}^{\infty} \int_{0}^{\vartheta} u_{1 z}(x, y, z, \tau) d \tau d \vartheta\right]\right\} \\
& -K(z) \frac{\beta(z)}{\rho(z)} \frac{\partial}{\partial z} \int_{0}^{\infty} \int_{0}^{\vartheta} T(x, y, z, \tau) d \tau d \vartheta+u_{0 z} .
\end{aligned}
$$

Framework this paper we determine concentration of dopant, concentrations of radiation defects and components of displacement vector by using the second-order approximation framework method of averaging of function corrections. This approximation is usually enough good approximation to make qualitative analysis and to obtain some quantitative results. All obtained results have been checked by comparison with results of numerical simulations.

\section{Discussion}

In this section, we analyzed dynamics of redistributions of dopant and radiation defects during annealing and under influence of mismatchinduced stress and modification of porosity. Typical distributions of concentrations of dopant in heterostructures are presented on Figures 2 and 3 for diffusion and ion types of doping, respectively. These distributions have been calculated for the case, when value of dopant diffusion coefficient in doped area is larger, than in nearest areas. The figures show, that inhomogeneity of heterostructure gives us possibility to increase compactness of concentrations of dopants and at the same time to increase homogeneity of dopant distribution in doped part of epitaxial layer. However framework this approach of manufacturing of bipolar 
transistor it is necessary to optimize annealing of dopant and/or radiation defects. Reason of this optimization is following. If annealing time is small, the dopant did not achieve any interfaces between materials of heterostructure. In this situation one cannot find any modifications of distribution of concentration of dopant. If annealing time is large, distribution of concentration of dopant is too homogeneous. We optimize annealing time framework recently introduces approach [12, 29-36]. Framework this criterion we approximate real distribution of concentration of dopant by step-wise function (see Figures 4 and 5). Farther we determine optimal values of annealing time by minimization of the following mean-squared error:

$$
U=\frac{1}{L_{x} L_{y} L_{z}} \int_{0}^{L_{x}} \int_{0}^{L_{y}} \int_{0}^{L_{z}}[C(x, y, z, \Theta)-\psi(x, y, z)] d z d y d x
$$

where $\psi(x, y, z)$ is the approximation function. Dependences of optimal values of annealing time on parameters are presented on Figures 6 and 7 for diffusion and ion types of doping, respectively. It should be noted, that it is necessary to anneal radiation defects after ion implantation. One could find spreading of concentration of distribution of dopant during this annealing. In the ideal case distribution of dopant achieves appropriate interfaces between materials of heterostructure during annealing of radiation defects. If dopant did not achieves any interfaces during annealing of radiation defects, it is practicably to additionally anneal the dopant. In this situation optimal value of additional annealing time of implanted dopant is smaller, than annealing time of infused dopant. 


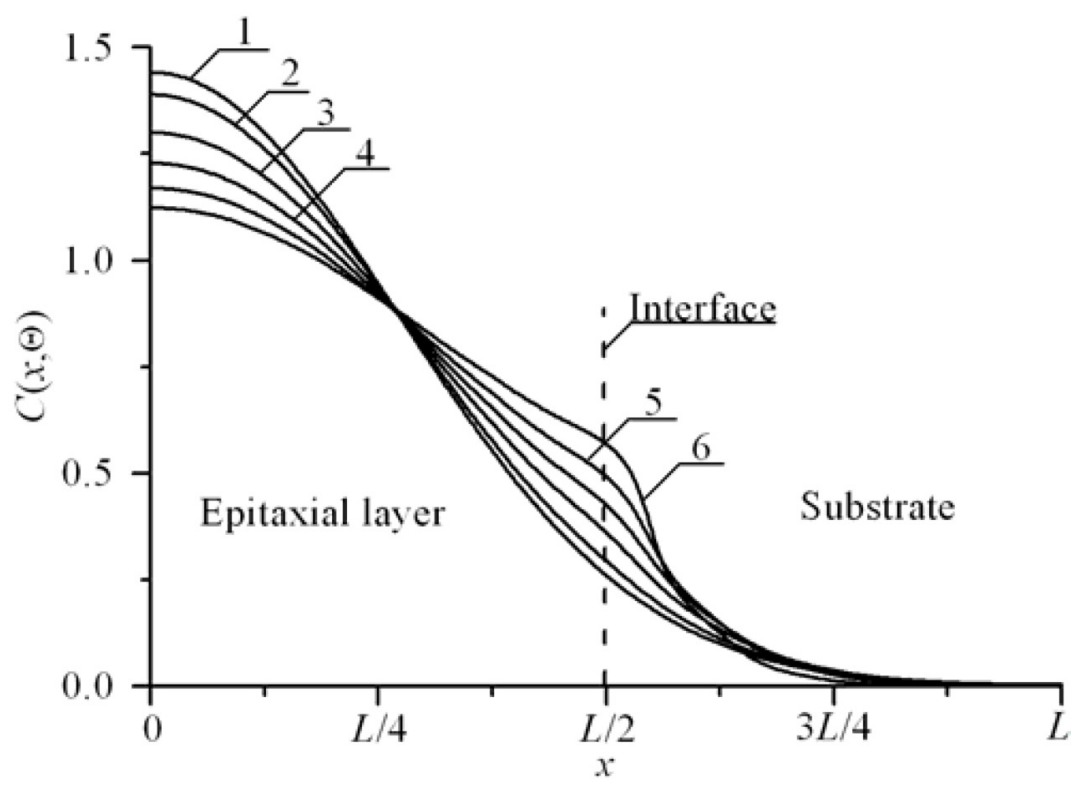

Figure 2. Distributions of concentration of infused dopant in heterostructure from Figure 1 in direction, which is perpendicular to interface between epitaxial layer substrate. Increasing of number of curve corresponds to increasing of difference between values of dopant diffusion coefficient in layers of heterostructure under condition, when value of dopant diffusion coefficient in epitaxial layer is larger, than value of dopant diffusion coefficient in substrate. 


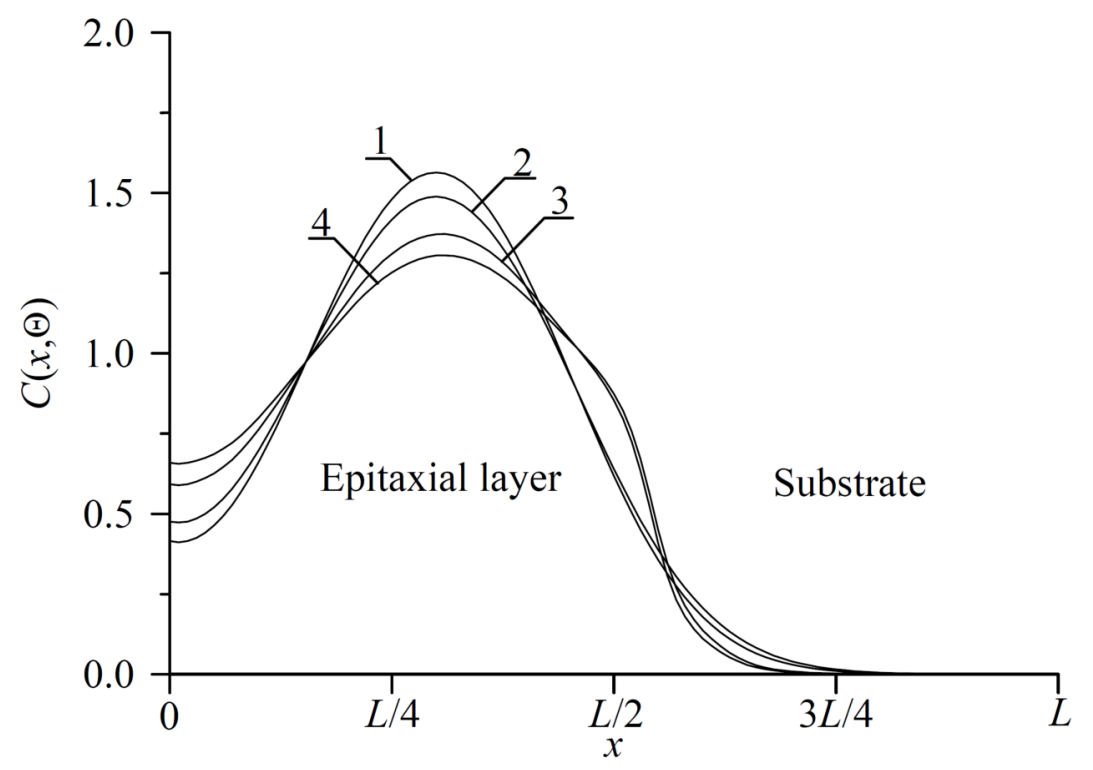

Figure 3. Distributions of concentration of implanted dopant in heterostructure from Figure 1 in direction, which is perpendicular to interface between epitaxial layer substrate. Curves 1 and 3 corresponds to annealing time $\Theta=0.0048\left(L_{x}^{2}+L_{y}^{2}+L_{z}^{2}\right) / D_{0}$. Curves 2 and 4 corresponds to annealing time $\Theta=0.0057\left(L_{x}^{2}+L_{y}^{2}+L_{z}^{2}\right) / D_{0}$. Curves 1 and 2 corresponds to homogeneous sample. Curves 3 and 4 corresponds to heterostructure under condition, when value of dopant diffusion coefficient in epitaxial layer is larger, than value of dopant diffusion coefficient in substrate. 


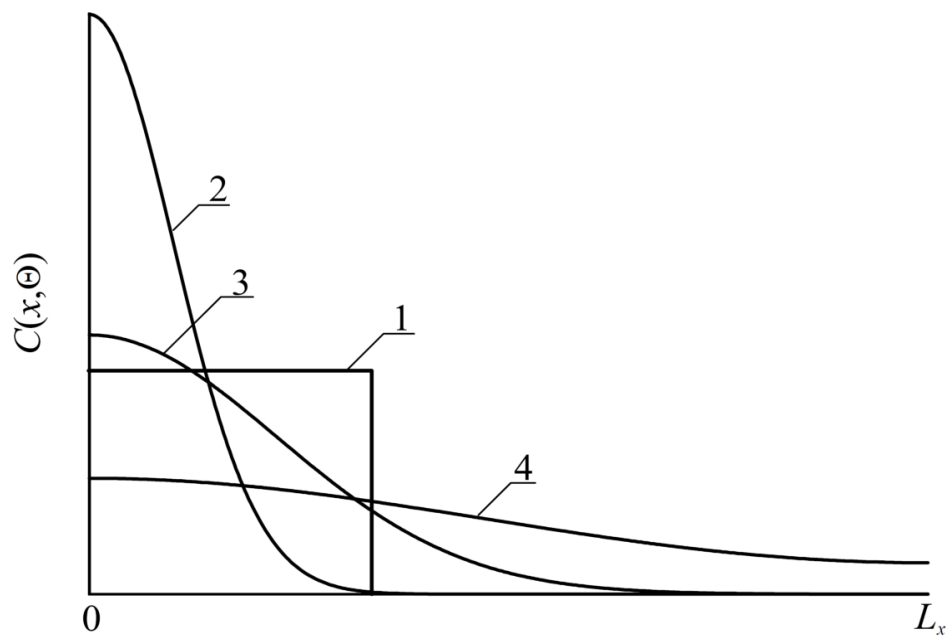

Figure 4. Spatial distributions of dopant in heterostructure after dopant infusion. Curve 1 is idealized distribution of dopant. Curves 2-4 are real distributions of dopant for different values of annealing time. Increasing of number of curve corresponds to increasing of annealing time.

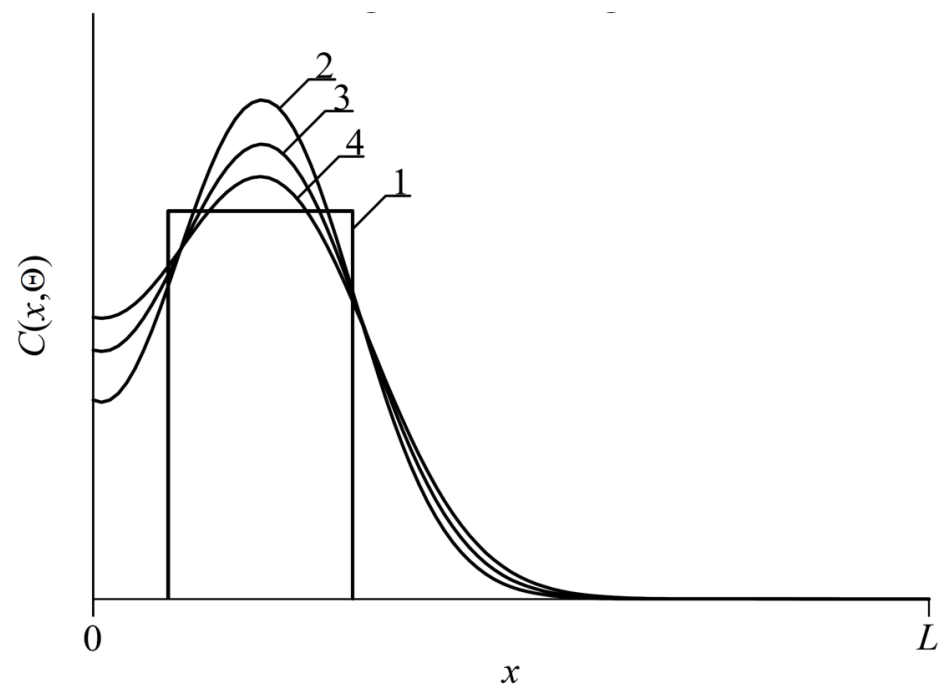

Figure 5. Spatial distributions of dopant in heterostructure after ion implantation. Curve 1 is idealized distribution of dopant. Curves 2-4 are real distributions of dopant for different values of annealing time. Increasing of number of curve corresponds to increasing of annealing time. 


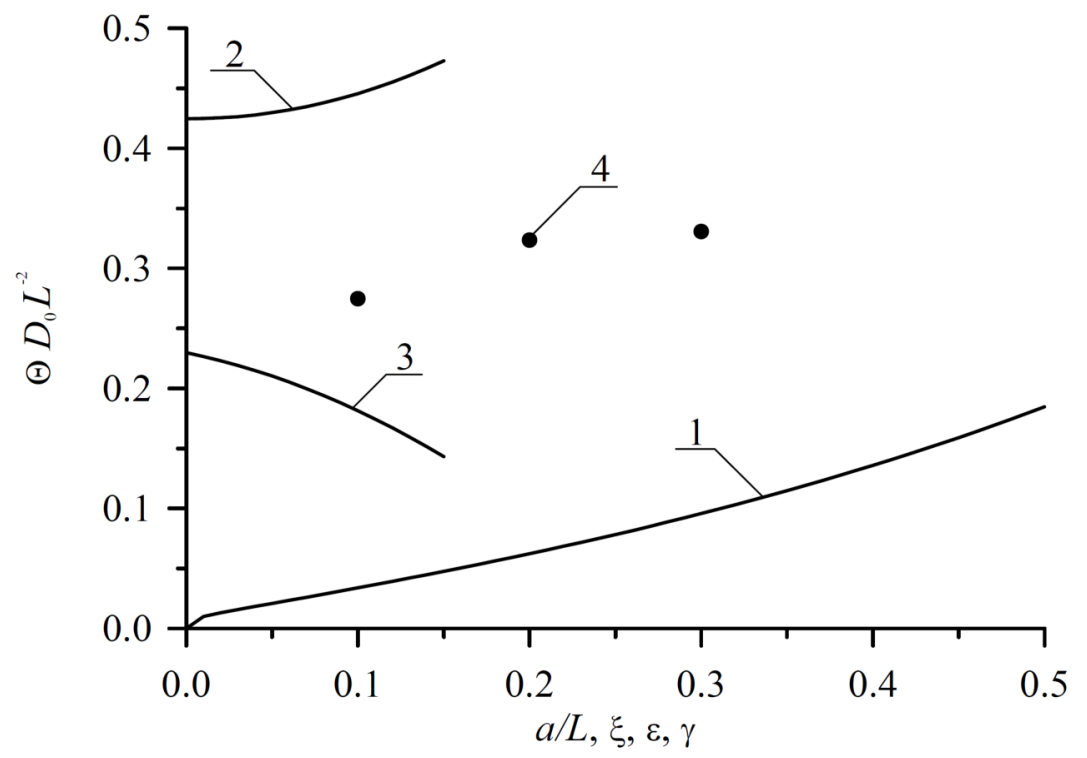

Figure 6. Dependences of dimensionless optimal annealing time for doping by diffusion, which have been obtained by minimization of meansquared error, on several parameters. Curve 1 is the dependence of dimensionless optimal annealing time on the relation $a / L$ and $\xi=\gamma=0$ for equal to each other values of dopant diffusion coefficient in all parts of heterostructure. Curve 2 is the dependence of dimensionless optimal annealing time on value of parameter $\varepsilon$ for $a / L=1 / 2$ and $\xi=\gamma=0$. Curve 3 is the dependence of dimensionless optimal annealing time on value of parameter $\xi$ for $a / L=1 / 2$ and $\varepsilon=\gamma=0$. Curve 4 is the dependence of dimensionless optimal annealing time on value of parameter $\gamma$ for $a / L=1 / 2$ and $\varepsilon=\xi=0$. 


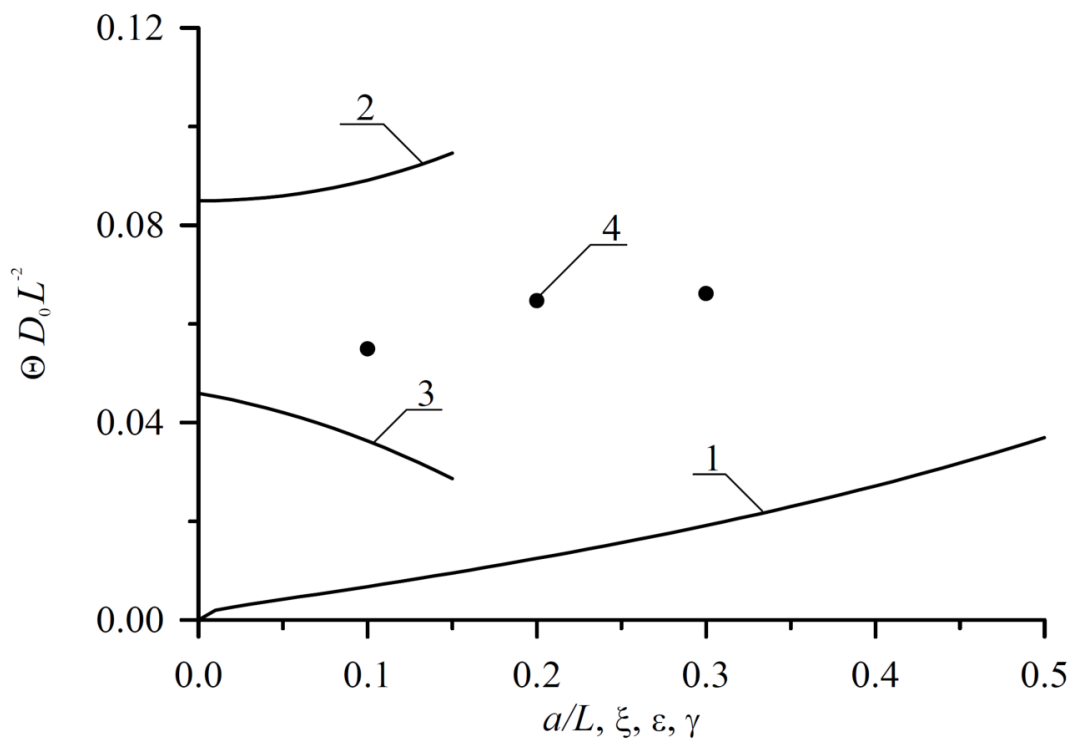

Figure 7. Dependences of dimensionless optimal annealing time for doping by ion implantation, which have been obtained by minimization of mean-squared error, on several parameters. Curve 1 is the dependence of dimensionless optimal annealing time on the relation $a / L$ and $\xi=\gamma=0$ for equal to each other values of dopant diffusion coefficient in all parts of heterostructure. Curve 2 is the dependence of dimensionless optimal annealing time on value of parameter $\varepsilon$ for $a / L=1 / 2$ and $\xi=\gamma=0$. Curve 3 is the dependence of dimensionless optimal annealing time on value of parameter $\xi$ for $a / L=1 / 2$ and $\varepsilon=\gamma=0$. Curve 4 is the dependence of dimensionless optimal annealing time on value of parameter $\gamma$ for $a / L=1 / 2$ and $\varepsilon=\xi=0$.

Farther we analyzed influence of relaxation of mechanical stress on distribution of dopant in doped areas of heterostructure. Under following condition $\varepsilon_{0}<0$, one can find compression of distribution of concentration of dopant near interface between materials of heterostructure. Contrary (at $\varepsilon_{0}>0$ ) one can find spreading of 
distribution of concentration of dopant in this area. This changing of distribution of concentration of dopant could be at least partially compensated by using laser annealing [36]. This type of annealing gives us possibility to accelerate diffusion of dopant and another processes in annealed area due to inhomogeneous distribution of temperature and Arrhenius law. Accounting relaxation of mismatch-induced stress in heterostructure could leads to changing of optimal values of annealing time. At the same time modification of porosity gives us possibility to decrease value of mechanical stress. On the one hand mismatch-induced stress could be used to increase density of elements of integrated circuits. On the other hand could leads to generation dislocations of the discrepancy. Figures 8 and 9 show distributions of concentration of vacancies in porous materials and component of displacement vector, which is perpendicular to interface between layers of heterostructure.

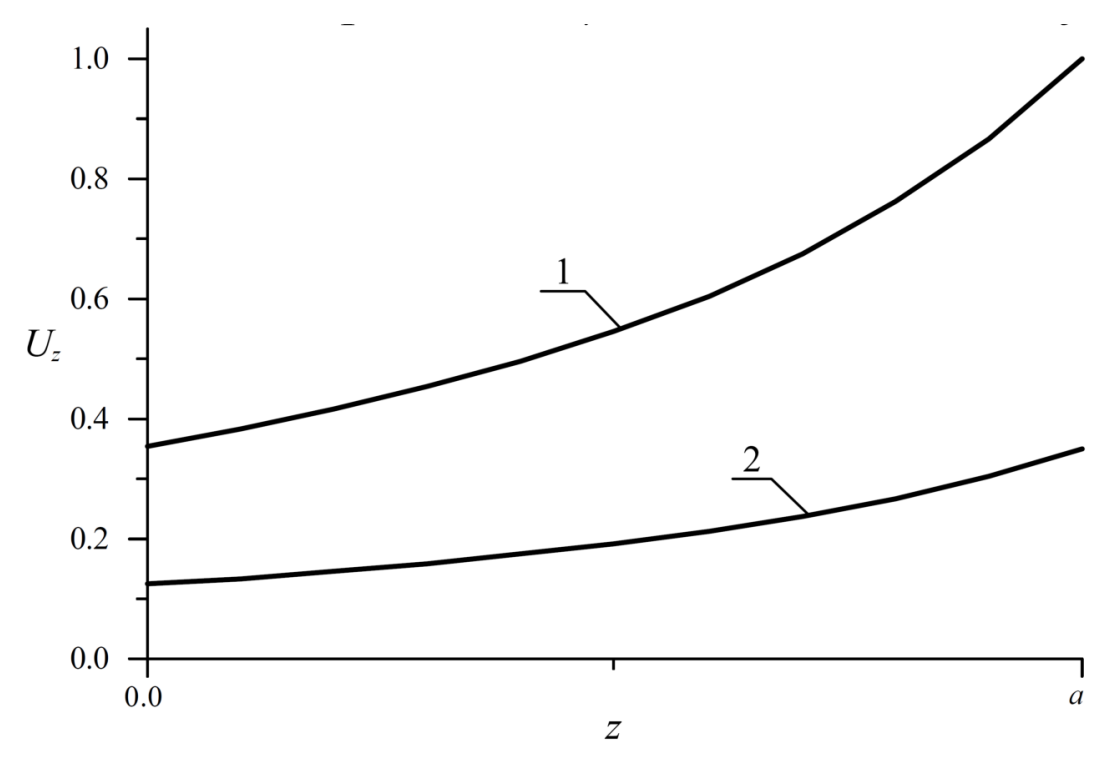

Figure 8. Normalized dependences of component $u_{z}$ of displacement vector on coordinate $z$ for nonporous (curve 1) and porous (curve 2) epitaxial layers. 


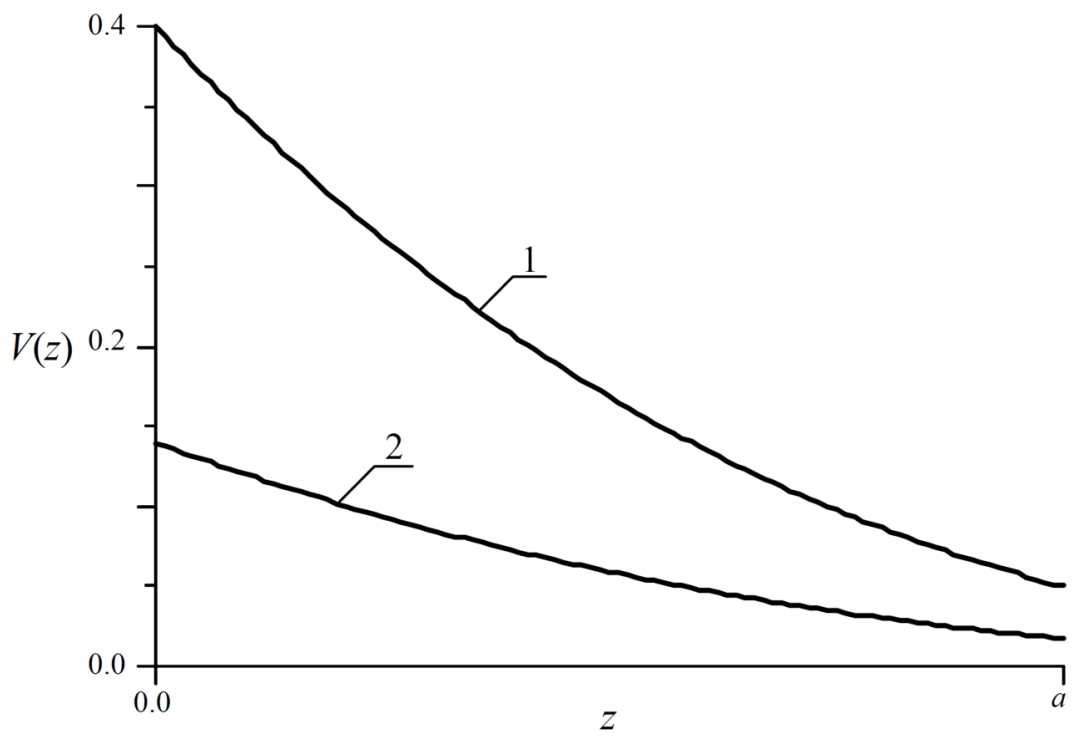

Figure 9. Normalized dependences of vacancy concentrations on coordinate $z$ in unstressed (curve 1) and stressed (curve 2) epitaxial layers.

\section{Conclusion}

In this paper, we model redistribution of infused and implanted dopants with account relaxation mismatch-induced stress during manufacturing field-effect heterotransistors framework a downconversion mixer circuit. We formulate recommendations for optimization of annealing to decrease dimensions of transistors and to increase their density. We formulate recommendations to decrease mismatch-induced stress. Analytical approach to model diffusion and ion types of doping with account concurrent changing of parameters in space and time has been introduced. At the same time the approach gives us possibility to take into account nonlinearity of considered processes. 


\section{References}

[1] V. I. Lachin and N. S. Savelov, Electronics, Rostov-on-Don: Phoenix 2001.

[2] A. Polishscuk, Modern Electronics, Issue 12 (2004), 8-11.

[3] G. Volovich, Modern Electronics, Issue 2 (2006), 10-17.

[4] A. Kerentsev and V. Lanin, Power Electronics, Issue 1 (2008), 34.

[5] A. O. Ageev, A. E. Belyaev, N. S. Boltovets, V. N. Ivanov, R. V. Konakova, Ya. Ya. Kudryk, P. M. Lytvyn, V. V. Milenin and A. V. Sachenko, Au-TiB $x-n-6 H-S i C$

Schottky barrier diodes: Specific features of charge transport in rectifying and nonrectifying contacts, Semiconductors 43(7) (2009), 865-871.

DOI: https://doi.org/10.1134/S1063782609070070

[6] Jung-Hui Tsai, Shao-Yen Chiu, Wen-Shiung Lour and Der-Feng Guo, Highperformance InGaP/GaAs pnp $\delta$-doped heterojunction bipolar transistor, Semiconductors 43(7) (2009), 939-942.

\section{DOI: https://doi.org/10.1134/S1063782609070227}

[7] O. V. Aleksandrov, A. O. Zakhar'in, N. A. Sobolev, E. I. Shek, M. M. Makoviichuk and E. O. Parshin, Formation of donor centers upon annealing of dysprosium-and holmium-implanted silicon, Semiconductors 32(9) (1998), 921-923.

DOI: https://doi.org/10.1134/1.1187515

[8] I. B. Ermolovich, V. V. Milenin, R. A. Red'ko and S. M. Red'ko, Specific features of recombination processes in CdTe films produced in different temperature conditions of growth and subsequent annealing, Semiconductors 43(8) (2009), 980-984.

DOI: https://doi.org/10.1134/S106378260908003X

[9] P. Sinsermsuksakul, K. Hartman, S. B. Kim, J. Heo, L. Sun, H. H. Park, R. Chakraborty, T. Buonassisi and R. G. Gordon, Enhancing the efficiency of SnS solar cells via band-offset engineering with a zinc oxysulfide buffer layer, Applied Physics Letters 102(5) (2013); Article 053901.

DOI: https://doi.org/10.1063/1.4789855

[10] J. G. Reynolds, C. L. Reynolds Jr., A. Mohanta, J. F. Muth, J. E. Rowe, H. O. Everitt and D. E. Aspnes, Shallow acceptor complexes in p-type ZnO, Applied Physics Letters 102(15) (2013); Article 152114.

DOI: https://doi.org/10.1063/1.4802753

[11] N. I. Volokobinskaya, I. N. Komarov, T. V. Matyukhina, V. I. Reshetnikov, A. A. Rush, I. V. Falina and A. S. Yastrebov, A study of technological processes in the production of high-power high-voltage bipolar transistors incorporating an array of inclusions in the collector region, Semiconductors 35(8) (2001), 974-978.

DOI: https://doi.org/10.1134/1.1393038 
[12] E. L. Pankratov and E. A. Bulaeva, Doping of materials during manufacture $p$ - $n$-junctions and bipolar transistors: Analytical approaches to model technological approaches and ways of optimization of distributions of Dopants, Reviews in Theoretical Science 1(1) (2013), 58-82.

DOI: https://doi.org/10.1166/rits.2013.1004

[13] S. A. Kukushkin, A. V. Osipov and A. I. Romanychev, Epitaxial growth of zinc oxide by the method of atomic layer deposition on $\mathrm{SiC} / \mathrm{Si}$ substrates, Physics of the Solid State 58(7) (2016), 1448-1452.

DOI: https://doi.org/10.1134/S1063783416070246

[14] E. M. Trukhanov, A. V. Kolesnikov and I. D. Loshkarev, Long-range stresses generated by misfit dislocations in epitaxial films, Russian Microelectronics 44(8) (2015), 552-558.

DOI: https://doi.org/10.1134/S1063739715080119

[15] E. L. Pankratov and E. A. Bulaeva, On optimization of regimes of epitaxy from gas phase: Some analytical approaches to model physical processes in reactors for epitaxy from gas phase during growth films, Reviews in Theoretical Science 3(4) (2015), 365-398.

DOI: https://doi.org/10.1166/rits.2015.1041

[16] K. K. Ong, K. L. Pey, P. S. Lee, A. T. S. Wee, X. C. Wang and Y. F. Chong, Dopant distribution in the recrystallization transient at the maximum melt depth induced by laser annealing, Applied Physics Letters 89(17) (2006); Article 172111.

DOI: https://doi.org/10.1063/1.2364834

[17] H. T. Wang, L. S. Tan and E. F. Chor, Pulsed laser annealing of Be-implanted GaN, Journal of Applied Physics 98(9) (2005); Article 094901.

DOI: https://doi.org/10.1063/1.2120893

[18] Yu. V. Bykov, A. G. Eremeev, N. A. Zharova, I. V. Plotnikov, K. I. Rybakov, M. N. Drozdov, Yu. N. Drozdov and V. D. Skupov, Diffusion processes in semiconductor structures during microwave annealing, Radiophysics and Quantum Electronics 46(9) (2003), 749-755.

DOI: https://doi.org/10.1023/B:RAQE.0000025008.97954.1c

[19] M. Tytgat, N. Van Thienen and P. Reynaert, A 90-GHz receiver in 40-nm CMOS for plastic waveguide links, Analog Integrated Circuits and Signal Processing 83(1) (2015), 55-64.

DOI: https://doi.org/10.1007/s10470-015-0510-6

[20] Y. W. Zhang and A. F. Bower, Numerical simulations of island formation in a coherent strained epitaxial thin film system, Journal of the Mechanics and Physics of Solids 47(11) (1999), 2273-2297.

DOI: https://doi.org/10.1016/S0022-5096(99)00026-5 
[21] L. D. Landau and E. M. Lefshits, Theoretical Physics, 7, Theory of Elasticity, Physmatlit, Moscow, 2001.

[22a] M. Kitayama, T. Narushima, W. C. Carter, R. M. Cannon and A. M. Glaeser, The Wulff shape of alumina, I: Modeling the kinetics of morphological evolution, Journal of the American Ceramic Society 83(10) (2000), 2561-2531.

DOI: https://doi.org/10.1111/j.1151-2916.2000.tb01591.x

[22b] M. Kitayama, T. Narushima and A. M. Glaeser, The Wulff shape of alumina, II: Experimental measurements of pore shape evolution rates, Journal of the American Ceramic Society 83(10) (2000), 2572-2583.

DOI: https://doi.org/10.1111/j.1151-2916.2000.tb01592.x

[23] P. G. Cheremskoy, V. V. Slesov and V. I. Betekhtin, Pore in Solid Bodies, Energoatomizdat, Moscow, 1990.

[24] Z. Yu. Gotra, Technology of Microelectronic Devices, Radio and Communication, Moscow, 1991.

[25] P. M. Fahey, P. B. Griffin and J. D. Plummer, Point defects and dopant diffusion in silicon, Reviews of Modern Physics 61(2) (1989); Article 289.

DOI: https://doi.org/10.1103/RevModPhys.61.289

[26] V. L. Vinetskiy and G. A. Kholodar, Radiative Physics of Semiconductors, Naukova Dumka, Kiev, 1979.

[27] M. G. Mynbaeva, E. N. Mokhov, A. A. Lavrent'ev and K. D. Mynbaev, Hightemperature diffusion doping of porous silicon carbide, Technical Physics Letters 34(9) (2008); Article 731.

DOI: https://doi.org/10.1134/S1063785008090034

[28] Yu. D. Sokolov, Applied Mechanics 1(1) (1955), 23-35.

[29] E. L. Pankratov, Dopant diffusion dynamics and optimal diffusion time as influenced by diffusion-coefficient nonuniformity, Russian Microelectronics 36(1) (2007), 33-39.

DOI: https://doi.org/10.1134/S1063739707010040

[30] E. L. Pankratov, Redistribution of a dopant during annealing of radiation defects in a multilayer structure by laser scans for production of an implanted-junction rectifier, International Journal of Nanoscience 7(4-5) (2008), 187-197.

DOI: https://doi.org/10.1142/S0219581X08005328

[31] E. L. Pankratov and E. A. Bulaeva, Decreasing of quantity of radiation defects in an implanted-junction rectifiers by using overlayers, International Journal of MicroNano Scale Transport 3(3-4) (2012), 119-130.

DOI: http://dx.doi.org/10.1260/1759-3093.3.3-4.119 
[32] E. L. Pankratov and E. A. Bulaeva Optimization of manufacturing of emittercoupled logic to decrease surface of chip, International Journal of Modern Physics B 29(5) (2015); Article 1550023.

DOI: https://doi.org/10.1142/S021797921550023X

[33] E. L. Pankratov, On approach to optimize manufacturing of bipolar heterotransistors framework circuit of an operational amplifier to increase their integration rate: Influence mismatch-induced stress, Journal of Computational and Theoretical Nanoscience 14(10) (2017), 4885-4899.

DOI: https://doi.org/10.1166/jctn.2017.6899

[34] E. L. Pankratov and E. A. Bulaeva, An approach to increase the integration rate of planar drift heterobipolar transistors, Materials Science in Semiconductor Processing 34 (2015), 260-268.

DOI: https://doi.org/10.1016/j.mssp.2015.02.054

[35] E. L. Pankratov and E. A. Bulaeva, An approach to manufacture a heterobipolar transistors in thin film structures: On the method of optimization, International Journal of Micro-Nano Scale Transport 4(1-2) (2013), 17-32.

DOI: http://dx.doi.org/10.1260/1759-3093.4.1-2.17

[36] E. L. Pankratov, Increasing of the sharpness of $p$ - $n$ junctions by Laser Pulses, Nano 6(1) (2011), 31-40.

DOI: https://doi.org/10.1142/S1793292011002329 